\title{
HIGHER ORDER RIESZ TRANSFORMS IN THE ULTRASPHERICAL SETTING AS PRINCIPAL VALUE INTEGRAL OPERATORS
}

\author{
JORGE J. BETANCOR, JUAN C. FARIÑA, LOURDES RODRÍGUEZ-MESA, AND RICARDO TESTONI

\begin{abstract}
In this paper we represent the $k$-th Riesz transform in the ultraspherical setting as a principal value integral operator for every $k \in \mathbb{N}$. We also measure the speed of convergence of the limit by proving $L^{p}$-boundedness properties for the oscillation and variation operators associated with the corresponding truncated operators.
\end{abstract}

\section{INTRODUCTION}

Muckenhoupt and Stein [10] introduced a notion of conjugate functions associated with ultraspherical expansions. In this setting the conjugate function appears as a boundary value of a conjugate harmonic extension associated with a suitable Cauchy-Riemann type equations.

Assume that $\lambda>0$. For every $n \in \mathbb{N}$, we denote by $P_{n}^{\lambda}$ the ultraspherical polynomial of degree $n([14])$. These polynomials are defined by the generating relation

$$
\left(1-2 t w+w^{2}\right)^{-\lambda}=\sum_{k=0}^{\infty} w^{k} P_{k}^{\lambda}(t) .
$$

The sequence $\left\{P_{n}^{\lambda}(\cos \theta)\right\}_{n \in \mathbb{N}}$ is orthogonal and complete in the space $L^{2}\left((0, \pi), d m_{\lambda}(\theta)\right)$, where $d m_{\lambda}(\theta)=(\sin \theta)^{2 \lambda} d \theta$. When $2 \lambda=k-2$, with $k \in \mathbb{N}$, the $\lambda$-ultraspherical polynomial $P_{n}^{\lambda}, n \in \mathbb{N}$, arises in the Fourier analysis of functions in the surface of the $n$-Euclidean space sphere that are invariant under the rotations leaving a given axis fixed.

For every $n \in \mathbb{N}, P_{n}^{\lambda}(\cos \theta)$ is an eigenfunction of the operator

$$
L_{\lambda}=-\frac{d^{2}}{d \theta^{2}}-2 \lambda \cot \theta \frac{d}{d \theta}+\lambda^{2}
$$

associated with the eigenvalue $\mu_{n}=(n+\lambda)^{2}$. The operator $L_{\lambda}$ can be written as follows

$$
L_{\lambda}=-\left(\frac{d}{d \theta}\right)^{*} \frac{d}{d \theta}+\lambda^{2}
$$

Date: November 4, 2018.

2000 Mathematics Subject Classification. 42C05 (primary), 42C15 (secondary).

This paper is partially supported by MTM2007/65609. 
where $\left(\frac{d}{d \theta}\right)^{*}=\frac{d}{d \theta}+2 \lambda \cot \theta$ denotes the formal adjoint of $\frac{d}{d \theta}$ in $L^{2}\left((0, \pi), d m_{\lambda}(\theta)\right)$.

In [1] Buraczewski, Martínez, Torrea and Urban defined a Riesz transform in the ultraspherical setting associated to $L_{\lambda}$. Note that this operator $L_{\lambda}$ is slightly different than the one considered by Muckenhoupt and Stein (see [10, p. 23]). In [1] the authors follow the ideas developed in the monography of Stein [13].

Suppose that $f \in L^{2}\left((0, \pi), d m_{\lambda}(\theta)\right)$. The ultraspherical expansion of $f$ is

$$
f(\theta)=\sum_{n=0}^{\infty} a_{n}^{\lambda}(f){\frac{P_{n}^{\lambda}(\cos \theta)}{\left\|P_{n}^{\lambda}(\cos \cdot)\right\|}}_{L^{2}\left((0, \pi), d m_{\lambda}(\theta)\right)},
$$

where, for every $n \in \mathbb{N}$,

$$
a_{n}^{\lambda}(f)=\int_{0}^{\pi} f(\theta) \frac{P_{n}^{\lambda}(\cos \theta)}{\left\|P_{n}^{\lambda}(\cos \cdot)\right\|_{L^{2}\left((0, \pi), d m_{\lambda}(\theta)\right)}} d m_{\lambda}(\theta) .
$$

The Poisson integral $P_{t}^{\lambda}(f), t>0$, is given by

$$
P_{t}^{\lambda}(f)(\theta)=e^{-t \sqrt{L_{\lambda}}} f(\theta)=\sum_{n=0}^{\infty} a_{n}^{\lambda}(f) e^{-t(n+\lambda)} \frac{P_{n}^{\lambda}(\cos \theta)}{\left\|P_{n}^{\lambda}(\cos \cdot)\right\|_{L^{2}\left((0, \pi), d m_{\lambda}(\theta)\right)}}, \quad t>0 .
$$

According to $[10,(2.12)]$ we can write

$$
P_{t}^{\lambda} f(\theta)=\int_{0}^{\pi} r^{\lambda} P_{\lambda}\left(e^{-t}, \theta, \varphi\right) f(\varphi) d m_{\lambda}(\varphi), \quad t>0
$$

where, for each $0<r<1$ and $\theta, \varphi \in(0, \pi)$,

$$
P_{\lambda}(r, \theta, \varphi)=\frac{\lambda}{\pi}\left(1-r^{2}\right) \int_{0}^{\pi} \frac{\sin ^{2 \lambda-1} t}{\left(1-2 r(\cos \theta \cos \varphi+\sin \theta \sin \varphi \cos t)+r^{2}\right)^{\lambda+1}} d t .
$$

The $L^{p}$-boundedness properties for these Poisson integrals and the corresponding maximal operator were established in [1, Theorem 2.4] (see also [10, Theorem 2]). For every $\alpha>0$, the fractional power $L_{\lambda}^{-\alpha}$ of the operator $L_{\lambda}$ is defined by

$$
L_{\lambda}^{-\alpha} f(\theta)=\frac{1}{\Gamma(2 \alpha)} \int_{0}^{\infty} e^{-t \sqrt{L_{\lambda}}} f(\theta) t^{2 \alpha-1} d t, \quad f \in L^{2}\left((0, \pi), d m_{\lambda}(\theta)\right) .
$$

By using (1) we get, for every $\alpha>0$ and $f \in L^{2}\left((0, \pi), d m_{\lambda}(\theta)\right)$,

$$
\begin{aligned}
L_{\lambda}^{-\alpha} f(\theta) & =\frac{1}{\Gamma(2 \alpha)} \int_{0}^{\infty} \int_{0}^{\pi} P^{\lambda}\left(e^{-t}, \theta, \varphi\right) f(\varphi) d m_{\lambda}(\varphi) t^{2 \alpha-1} d t \\
& =\int_{0}^{\pi} f(\varphi) \frac{1}{\Gamma(2 \alpha)} \int_{0}^{1} P^{\lambda}(r, \theta, \varphi)\left(\log \frac{1}{r}\right)^{2 \alpha-1} \frac{1}{r} d r d m_{\lambda}(\varphi) .
\end{aligned}
$$

Following [13] the Riesz transform of order $k \in \mathbb{N}, R_{\lambda}^{k}$, is defined as

$$
R_{\lambda}^{k} f=\frac{d^{k}}{d \theta^{k}} L_{\lambda}^{-\frac{k}{2}} f
$$


when $f$ is a nice function (for instance, $f \in \operatorname{span}\left\{P_{n}^{\lambda}(\cos \theta)\right\}_{n \in \mathbb{N}}$ or $f$ is a smooth function with compact support on $(0, \pi))$.

It was proved in [1, Theorem 2.14] (when $k=1$ ) and [2, Theorem 1.4] (when $k>1$ ) that the operator $R_{\lambda}^{k}$ can be extended to $L^{p}\left((0, \pi), w(\theta) d m_{\lambda}(\theta)\right)$ as a bounded operator from $L^{p}\left((0, \pi), w(\theta) d m_{\lambda}(\theta)\right)$ into itself, when $1<p<\infty$ and $w \in A_{\lambda}^{p}$, and as a bounded operator from $L^{1}\left((0, \pi), w(\theta) d m_{\lambda}(\theta)\right)$ into $L^{1, \infty}\left((0, \pi), w(\theta) d m_{\lambda}(\theta)\right)$, when $w \in A_{\lambda}^{1}$. Here, for every $1 \leq p<\infty$, by $A_{\lambda}^{p}$ we denote the Muckenhoupt class of weights associated with the doubling measure $d m_{\lambda}(\theta)$ on $(0, \pi)$.

In this paper we prove that the $k$-th Riesz transform $R_{\lambda}^{k}$ is a principal value integral operator, for every $k \in \mathbb{N}$. We extend [1, Theorem 2.13] where the result is shown for $k=1$.

Theorem 1.1. Let $\lambda>0$ and $k \in \mathbb{N}$. For every $1 \leq p<\infty$ and $\omega \in A_{\lambda}^{p}$, we have that if $f \in L^{p}\left((0, \pi), w(\theta) d m_{\lambda}(\theta)\right)$

$$
R_{\lambda}^{k} f(\theta)=\lim _{\varepsilon \rightarrow 0^{+}} \int_{0,|\theta-\varphi|>\varepsilon}^{\pi} R_{\lambda}^{k}(\theta, \varphi) f(\varphi) d m_{\lambda}(\varphi)+\gamma_{k} f(\theta), \quad \text { a.e. } \theta \in(0, \pi)
$$

where

$$
R_{\lambda}^{k}(\theta, \varphi)=\frac{1}{\Gamma(k)} \int_{0}^{1} \frac{\partial^{k}}{\partial \theta^{k}} P_{\lambda}(r, \theta, \varphi)\left(\log \frac{1}{r}\right)^{k-1} r^{\lambda-1} d r, \quad \theta, \varphi \in(0, \pi),
$$

and $\gamma_{k}=0$, when $k$ is odd, and $\gamma_{k}=(-1)^{\frac{k}{2}}$, when $k$ is even.

The complete proof of this theorem is presented in Section 2. It is a crucial point in the proof the estimates established in Lemma 2.1 below. We prove in this lemma that in the local region, that is, close to the diagonal $\{\theta=\varphi\}$, the kernel $R_{\lambda}^{k}(\theta, \varphi)$ differs from the kernel of the $k$-th Euclidean Riesz transform by an integrable function. Also, we show that far from the diagonal $R_{\lambda}^{k}(\theta, \varphi)$ is bounded by Hardy type kernels.

Suppose that $\left\{T_{\varepsilon}\right\}_{\varepsilon>0}$ is a family of operators defined on $L^{p}(\Omega, \mu)$, for some measure space $(\Omega, \mu)$ and $1 \leq p<\infty$, such that for every $f \in L^{p}(\Omega, \mu)$ there exists $\lim _{\varepsilon \rightarrow 0^{+}} T_{\varepsilon} f(x)$, $\mu$-a.e. $x \in \Omega$. It is an interesting question to measure the speed of that convergence. In order to do this it is usual to analyze expressions involving differences like $\left|T_{\varepsilon} f-T_{\eta} f\right|, \varepsilon, \eta>0$. The oscillation and variation operators defined as follows have been used for this purpose. The oscillation operator associated with $\left\{T_{\varepsilon}\right\}_{\varepsilon>0}$ is defined by

$$
O\left(\left\{T_{\varepsilon}\right\}\right)(f)(x)=\left(\sum_{i=0}^{\infty} \sup _{t_{i+1} \leq \varepsilon_{i+1}<\varepsilon_{i}<t_{i}}\left|T_{\varepsilon_{i+1}} f(x)-T_{\varepsilon_{i}} f(x)\right|^{2}\right)^{\frac{1}{2}},
$$


for a fixed real sequence $\left\{t_{i}\right\}_{i \in \mathbb{N}}$ decreasing to zero. For every $\rho>2$ the $\rho$-variation operator for $\left\{T_{\varepsilon}\right\}_{\varepsilon>0}$ is given as follows

$$
V_{\rho}\left(\left\{T_{\varepsilon}\right\}\right)(f)(x)=\sup _{\left\{\varepsilon_{i}\right\}_{i \in \mathbb{N}}}\left(\sum_{i=0}^{\infty}\left|T_{\varepsilon_{i+1}} f(x)-T_{\varepsilon_{i}} f(x)\right|^{\rho}\right)^{\frac{1}{\rho}},
$$

where the supremum is taken over all real sequences $\left\{\varepsilon_{i}\right\}_{i \in \mathbb{N}}$ decreasing to zero. These operators appear in an ergodic context. In [3], [4] and [8] the $L^{p}$-boundedness properties for the oscillation and variation operators were studied when $T_{\varepsilon}, \varepsilon>0$, represents the truncated Hilbert and higher dimensional Riesz transform, and Euclidean Poisson semigroup (see also [6] and the references therein). The corresponding results for the truncated ultraspherical Riesz transform were established in [1, Theorem 8.3]. We also measure the speed of convergence in (2) in terms of variation and oscillation operators for the corresponding truncated operators. Next result is an extension of [1, Theorem 8.3] for the higher Riesz transform $R_{\lambda}^{k}$.

Theorem 1.2. Let $\lambda>0$ and $k \in \mathbb{N}$. For every $\varepsilon>0$ we define by $R_{\lambda, \varepsilon}^{k}$ the $\varepsilon$-truncation of $R_{\lambda}^{k}$ as follows

$$
R_{\lambda, \varepsilon}^{k}(f)(\theta)=\int_{0,|\theta-\varphi|>\varepsilon}^{\pi} R_{\lambda}^{k}(\theta, \varphi) f(\varphi) d m_{\lambda}(\varphi) .
$$

If $\left\{t_{i}\right\}_{i \in \mathbb{N}}$ is a real decreasing sequence that converges to zero, the oscillation operator $O\left(\left\{R_{\lambda, \varepsilon}^{k}\right\}\right)$ is a bounded operator from $L^{p}\left((0, \pi), d m_{\lambda}(\varphi)\right)$ into itself, for every $1<p<\infty$, and from $L^{1}\left((0, \pi), d m_{\lambda}(\varphi)\right)$ to $L^{1, \infty}\left((0, \pi), d m_{\lambda}(\varphi)\right)$. Also, for every $\rho>2$, the variation operator $V_{\rho}\left(\left\{R_{\lambda, \varepsilon}^{k}\right\}\right)$ is bounded from $L^{p}\left((0, \pi), d m_{\lambda}(\varphi)\right)$ into itself, for every $1<p<\infty$, and from $L^{1}\left((0, \pi), d m_{\lambda}(\varphi)\right)$ to $L^{1, \infty}\left((0, \pi), d m_{\lambda}(\varphi)\right)$.

We remark that the representation of the $k$-th Riesz transform $R_{\lambda}^{k}$ as a principal value integral operator will allow us to investigate weighted norm inequalities for $R_{\lambda}^{k}$ involving a class of weights wider than the Muckenhoupt class considered in [2]. This question will be studied in a forthcoming paper.

Troughout this paper by $C$ we always denote a positive constant that can change from one line to the other one and $i, j$ represent nonnegative integers.

\section{Proof of Theorem 1.1}

In [2, Theorem 1.5] it was established that, for every $k \in \mathbb{N}$, the $\mathrm{k}$-th Riesz transform $R_{\lambda}^{k}$ is a Calderón-Zygmund operator in the homogeneous type space $\left((0, \pi),||,. d m_{\lambda}(\theta)\right)$. Then, 
according to $\left[7\right.$, Theorem 9.4.5] the maximal operator $R_{\lambda, *}^{k}$ given by

$$
R_{\lambda, *}^{k}(f)=\sup _{\varepsilon>0}\left|R_{\lambda, \varepsilon}^{k}(f)\right|,
$$

where $R_{\lambda, \varepsilon}^{k}$ is defined as in Theorem 1.2, is bounded from $L^{p}\left((0, \pi), w(\theta) d m_{\lambda}(\theta)\right)$ into itself, when $1<p<\infty$ and $w \in A_{\lambda}^{p}$, and from $L^{1}\left((0, \pi), w(\theta) d m_{\lambda}(\theta)\right)$ into $L^{1, \infty}\left((0, \pi), w(\theta) d m_{\lambda}(\theta)\right)$, when $w \in A_{\lambda}^{1}$. Suppose we have proved that, for every $f \in C_{c}^{\infty}(0, \pi)$, there exists the limit

$$
T_{\lambda}^{k}(f)(\theta)=\lim _{\varepsilon \rightarrow 0^{+}} \int_{0,|\theta-\varphi|>\varepsilon}^{\pi} R_{\lambda}^{k}(\theta, \varphi) f(\varphi) d m_{\lambda}(\varphi), \quad \text { a.e. } \theta \in(0, \pi),
$$

and that $T_{\lambda}^{k} f=R_{\lambda}^{k} f-\gamma_{k} f$. Then, $L^{p}$-boundedness properties of the maximal operator $R_{\lambda, *}^{k}$ imply that the limit in (3) exists for almost all $\theta \in(0, \pi)$, for every $f \in L^{p}\left((0, \pi), w(\theta) d m_{\lambda}(\theta)\right)$, $1 \leq p<\infty$, and $w \in A_{\lambda}^{p}$. Moreover, by defining $T_{\lambda}^{k}$ in the obvious way on $L^{p}\left((0, \pi), w(\theta) d m_{\lambda}(\theta)\right)$, $1 \leq p<\infty, T_{\lambda}^{k}$ is a bounded operator from $L^{p}\left((0, \pi), w(\theta) d m_{\lambda}(\theta)\right)$ into itself, when $1<p<\infty$ and $w \in A_{\lambda}^{p}$, and from $L^{1}\left((0, \pi), w(\theta) d m_{\lambda}(\theta)\right)$ into $L^{1, \infty}\left((0, \pi), w(\theta) d m_{\lambda}(\theta)\right)$, when $w \in A_{\lambda}^{1}$. Hence, by [2, Theorem 1.4], we conclude that, for each $f \in L^{p}\left((0, \pi), w(\theta) d m_{\lambda}(\theta)\right), 1 \leq p<\infty$,

$$
R_{\lambda}^{k}(f)(\theta)=\lim _{\varepsilon \rightarrow 0^{+}} \int_{0,|\theta-\varphi|>\varepsilon}^{\pi} R_{\lambda}^{k}(\theta, \varphi) f(\varphi) d m_{\lambda}(\varphi)+\gamma_{k} f(\theta), \quad \text { a.e. } \theta \in(0, \pi),
$$

and the proof of this theorem would be finished.

Let now $f \in C_{c}^{\infty}(0, \pi)$ and $k \in \mathbb{N}$. We can write

$$
L_{\lambda}^{-\frac{k}{2}} f(\theta)=\sum_{n=0}^{\infty}(n+\lambda)^{-k} a_{n}^{\lambda}(f){\frac{P_{n}^{\lambda}(\cos \theta)}{\left\|P_{n}^{\lambda}(\cos \cdot)\right\|_{L^{2}\left((0, \pi), d m_{\lambda}(\theta)\right)}}} \quad \theta \in(0, \pi) .
$$

Then, since $f \in C_{c}^{\infty}(0, \pi), L_{\lambda}^{-\frac{k}{2}} f \in C^{\infty}(0, \pi)$ (see $[9,(2.4)$ and $(2.6)]$ ). We will see that

$$
\frac{d^{k}}{d \theta^{k}} L_{\lambda}^{-\frac{k}{2}} f(\theta)=\lim _{\varepsilon \rightarrow 0^{+}} \int_{0,|\theta-\varphi|>\varepsilon}^{\pi} f(\varphi) R_{\lambda}^{k}(\theta, \varphi) d m_{\lambda}(\varphi)+\gamma_{k} f(\theta), \quad \text { a.e. } \theta \in(0, \pi),
$$

where

$$
\begin{gathered}
R_{\lambda}^{k}(\theta, \varphi)=\frac{\partial^{k}}{\partial \theta^{k}}\left(\frac{1}{\Gamma(k)} \int_{0}^{1} r^{\lambda-1}\left(\log \frac{1}{r}\right)^{k-1} P_{\lambda}(r, \theta, \varphi) d r\right), \quad \theta, \varphi \in(0, \pi), \\
P_{\lambda}(r, \theta, \varphi)=\frac{\lambda}{\pi} \int_{0}^{\pi} \frac{\left(1-r^{2}\right)(\sin t)^{2 \lambda-1}}{\left(1-2 r(\cos \theta \cos \varphi+\sin \theta \sin \varphi \cos t)+r^{2}\right)^{\lambda+1}} d t, \quad r \in(0,1), \theta, \varphi \in(0, \pi),
\end{gathered}
$$

and $\gamma_{k}=0$, when $k$ is odd, and $\gamma_{k}=(-1)^{\frac{k}{2}}$, when $k$ is even. 
As in [2] we introduce the following useful notation:

$$
\begin{aligned}
& \sigma=\sin \theta \sin \varphi, \quad a=\cos \theta \cos \varphi+\sigma \cos t=\cos (\theta-\varphi)-\sigma(1-\cos t), \\
& b=\frac{\partial}{\partial \theta} a=-\sin \theta \cos \varphi+\cos \theta \sin \varphi \cos t=-\sin (\theta-\varphi)-\cos \theta \sin \varphi(1-\cos t), \\
& \Delta_{r}=1-2 r \cos (\theta-\varphi)+r^{2}=(1-r)^{2}+2 r(1-\cos (\theta-\varphi)), \Delta=\Delta_{1}, \\
& D_{r}=1-2 r a+r^{2}=\Delta_{r}+2 r \sigma(1-\cos t) .
\end{aligned}
$$

We divide the proof in several steps.

Step 1. We prove in the following that for every $\ell=0, \ldots, k-1$,

$$
\frac{d^{\ell}}{d \theta^{\ell}} L_{\lambda}^{-\frac{k}{2}} f(\theta)=\int_{0}^{\pi} f(\varphi) R_{\lambda}^{k, \ell}(\theta, \varphi) d m_{\lambda}(\varphi), \quad \theta \in(0, \pi)
$$

being

$$
R_{\lambda}^{k, \ell}(\theta, \varphi)=\frac{1}{\Gamma(k)} \int_{0}^{1} r^{\lambda-1}\left(\log \frac{1}{r}\right)^{k-1} \frac{\partial^{\ell}}{\partial \theta^{\ell}} P_{\lambda}(r, \theta, \varphi) d r, \quad \theta, \varphi \in(0, \pi) .
$$

Let $\ell \in \mathbb{N}, 0 \leq \ell \leq k-1$. Our objective is to establish that

$$
\left|R_{\lambda}^{k, \ell}(\theta, \varphi)\right| \leq C \begin{cases}(\sin \varphi)^{-2 \lambda-1}, & (\theta, \varphi) \in A_{1} ; \\ \frac{1}{(\sin \theta \sin \varphi)^{\lambda} \sqrt{|\theta-\varphi|},} & (\theta, \varphi) \in A_{2}, \theta \neq \varphi ; \\ (\sin \theta)^{-2 \lambda-1}, & (\theta, \varphi) \in A_{3} ;\end{cases}
$$

where $A_{i}, i=1,2,3$, are the sets in the next figure:

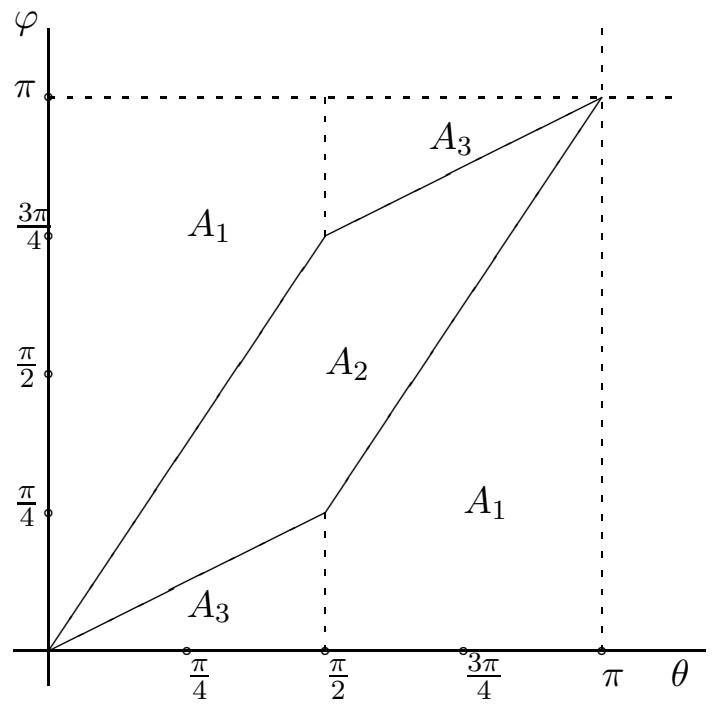

Figure 1 
According to [2, Lemma 3.5] we have that

$$
\frac{\partial^{\ell}}{\partial \theta^{\ell}}\left(\frac{1}{D_{r}^{\lambda+1}}\right)=\sum_{s, i, j} c_{\ell, s, i, j} \frac{r^{i+j} a^{i} b^{j}}{D_{r}^{\lambda+1+s}}
$$

where $c_{\ell, s, i, j} \neq 0$ only if

$$
s=1, \ldots, \ell, j \geq 2 s-\ell, \text { and } i+j=s .
$$

Moreover, by using the di Faà di Bruno's formula ([11, Theorem 2]), we can see that, for every $\ell \in \mathbb{N}, s=1, \ldots, \ell$, and $i+j=s$,

$$
c_{\ell, s, i, j}=2^{s} \ell ! s ! \sum \frac{(-1)^{s+j+\alpha\left(k_{1}, \ldots, k_{\ell}\right)}}{k_{1} ! \cdots k_{\ell} ! 1 !^{k_{1}} 2 !^{k_{2}} \cdots \ell !^{k_{\ell}}},
$$

where the sum is over all different solutions in nonnegative integers $k_{1}, \ldots, k_{\ell}$ of the system

$$
\begin{aligned}
& k_{1}+k_{2}+\cdots+k_{\ell}=s \\
& k_{1}+2 k_{2}+\ldots+\ell k_{\ell}=\ell \\
& \sum_{r \text { par }} k_{r}=i \\
& \sum_{r \text { impar }} k_{r}=j
\end{aligned}
$$

and

$$
\alpha\left(k_{1}, \ldots, k_{\ell}\right)=\sum_{r=2}^{\left[\frac{\ell}{2}\right]}(r-1)\left(k_{2 r-1}+k_{2 r}\right)+m_{\ell},
$$

being $m_{\ell}=0$, if $\ell$ is even, and $m_{\ell}=\frac{(\ell-1) k_{\ell}}{2}$, when $\ell$ is odd.

We define, for every $s, i, j$ satisfying (7),

$$
M_{\ell, s, i, j}(\theta, \varphi)=\int_{0}^{1} \int_{0}^{\pi} r^{i+j+\lambda-1}\left(\log \frac{1}{r}\right)^{k-1}\left(1-r^{2}\right) \frac{a^{i} b^{j}(\sin t)^{2 \lambda-1}}{D_{r}^{\lambda+1+s}} d t d r, \quad \theta, \varphi \in(0, \pi) .
$$

In order to obtain (5) it is then sufficient to see that

$$
\left|M_{\ell, s, i, j}(\theta, \varphi)\right| \leq C \begin{cases}(\sin \varphi)^{-2 \lambda-1}, & (\theta, \varphi) \in A_{1} ; \\ \frac{1}{(\sin \theta \sin \varphi)^{\lambda} \sqrt{|\theta-\varphi|},} & (\theta, \varphi) \in A_{2}, \theta \neq \varphi \\ (\sin \theta)^{-2 \lambda-1}, & (\theta, \varphi) \in A_{3}\end{cases}
$$

for each $s, i, j$ satisfying (7). 
Moreover, by the symmetry of the Figure 1 and since

$$
M_{\ell, s, i, j}(\pi-\theta, \pi-\varphi)=(-1)^{j} M_{\ell, s, i, j}(\theta, \varphi), \quad \theta, \varphi \in(0, \pi)
$$

when $s, i, j$ are as in $(7)$, we can assume that $(\theta, \varphi) \in\left(0, \frac{\pi}{2}\right) \times(0, \pi)$.

Let us fix $s, i, j$ verifying $(7), \theta \in\left(0, \frac{\pi}{2}\right)$ and $\varphi \in(0, \pi)$. By proceeding as in [2, Lemma 3.6], and using that $\log \frac{1}{r} \sim 1-r$, as $r \rightarrow 1^{-}$, and $D_{r} \geq C, r \in\left(0, \frac{1}{2}\right)$, we get

$$
\begin{aligned}
\left|M_{\ell, s, i, j}(\theta, \varphi)\right| & \leq\left(\int_{0}^{\frac{1}{2}} \int_{0}^{\pi}+\int_{\frac{1}{2}}^{1} \int_{0}^{\pi}\right) r^{i+j+\lambda-1}\left(\log \frac{1}{r}\right)^{k-1}\left(1-r^{2}\right) \frac{|a|^{i}|b|^{j}(\sin t)^{2 \lambda-1}}{D_{r}^{\lambda+1+s}} d t d r \\
& \leq C\left(\int_{0}^{\frac{1}{2}} r^{\lambda-1}\left(\log \frac{1}{r}\right)^{k-1} d r+\int_{\frac{1}{2}}^{1} \int_{0}^{\pi}(1-r)^{k} \frac{|b|^{j}(\sin t)^{2 \lambda-1}}{D_{r}^{\lambda+1+s}} d t d r\right) \\
& \leq C\left(1+\int_{\frac{1}{2}}^{1} \int_{0}^{\pi}(1-r)^{k} \frac{|b|^{j}(\sin t)^{2 \lambda-1}}{D_{r}^{\lambda+1+s}} d t d r\right) .
\end{aligned}
$$

It can be seen that, if $(\alpha, \beta) \in[0, \pi] \times[0, \pi], z \in(0,1)$ and $\alpha \leq z \beta$, then there exists $C>0$ such that $\sin (\beta-\alpha) \geq \min \{\sin \beta, \sin ((1-z) \beta)\} \geq C \sin \beta$, and that, if $(\alpha, \beta) \in[0, \pi / 2] \times[0, \pi]$ and $\frac{\alpha}{2} \leq \beta \leq \frac{3 \alpha}{2}$, then $\sin |\beta-\alpha| \leq \sin \alpha$ and $\sin \alpha \sim \sin \beta$. These considerations allow us to write

$$
|b|^{j} \leq C\left(|\sin (\theta-\varphi)|^{j}+(\sin \varphi)^{j}\right) \leq C \begin{cases}|\sin (\theta-\varphi)|^{j}, & \varphi \leq \frac{\theta}{2} \text { or } \varphi \geq \frac{3 \theta}{2} \\ (\sin \varphi)^{j}, & \frac{\theta}{2} \leq \varphi \leq \frac{3 \theta}{2}\end{cases}
$$

Then, since $1-\cos \alpha \geq(\sin \alpha)^{2} / \pi, \alpha \in[0, \pi]$, we obtain, when $\varphi \leq \frac{\theta}{2}$ or $\varphi \geq \frac{3 \theta}{2}$,

$$
\begin{aligned}
& \left|M_{\ell, s, i, j}(\theta, \varphi)\right| \leq C\left(1+(\sin |\theta-\varphi|)^{j} \int_{\frac{1}{2}}^{1} \frac{(1-r)^{k}}{\Delta_{r}^{\lambda+s+1}} d r\right) \\
& \leq C\left(1+(\sin |\theta-\varphi|)^{j} \int_{\frac{1}{2}}^{1} \frac{(1-r)^{2 s-j}}{\left(\Delta+(1-r)^{2}\right)^{\lambda+s+1}} d r\right) \\
& \leq C\left(1+\frac{(\sin |\theta-\varphi|)^{j}}{\Delta^{\lambda+\frac{j+1}{2}}} \int_{0}^{\frac{1}{2 \sqrt{\Delta}}} \frac{u^{2 s-j}}{\left(1+u^{2}\right)^{\lambda+s+1}} d u\right) \\
& \leq C \frac{1}{(\sin |\theta-\varphi|)^{2 \lambda+1}} \\
& \leq C \begin{cases}(\sin \varphi)^{-2 \lambda-1}, & \varphi \geq \frac{3 \theta}{2}, \\
(\sin \theta)^{-2 \lambda-1}, & \varphi \leq \frac{\theta}{2} .\end{cases}
\end{aligned}
$$


Suppose now that $\frac{\theta}{2} \leq \varphi \leq \frac{3 \theta}{2}, \theta \neq \varphi$. One can write

$$
\begin{aligned}
\int_{\frac{1}{2}}^{1} \int_{0}^{\pi}(1-r)^{k} \frac{|b|^{j}(\sin t)^{2 \lambda-1}}{D_{r}^{\lambda+1+s}} & d t d r \\
\leq & C\left(\int_{\frac{1}{2}}^{1} \int_{0}^{\pi}(1-r)^{k} \frac{(\sin |\theta-\varphi|)^{j}(\sin t)^{2 \lambda-1}}{D_{r}^{\lambda+1+s}} d t d r\right. \\
& +\int_{\frac{1}{2}}^{1} \int_{0}^{\frac{\pi}{2}}(1-r)^{k} \frac{(\sin \varphi(1-\cos t))^{j}(\sin t)^{2 \lambda-1}}{D_{r}^{\lambda+1+s}} d t d r \\
& \left.+\int_{\frac{1}{2}}^{1} \int_{\frac{\pi}{2}}^{\pi}(1-r)^{k} \frac{(\sin \varphi)^{j}(\sin t)^{2 \lambda-1}}{D_{r}^{\lambda+1+s}} d t d r\right)=\sum_{\beta=1}^{3} I_{\beta}(\theta, \varphi) .
\end{aligned}
$$

We analyze the first integral. By making two changes of variables, as in [2, p. 1235], and by taking into account that $2 s-j \leq \ell \leq k-1$ and that $\Delta=2(1-\cos (\theta-\varphi)) \sim(\sin |\theta-\varphi|)^{2} \sim$ $(\theta-\varphi)^{2}$ we get

$$
\begin{aligned}
I_{1}(\theta, \varphi) & \leq C(\sin |\theta-\varphi|)^{j} \int_{\frac{1}{2}}^{1}(1-r)^{k} \int_{0}^{\pi} \frac{(\sin t)^{2 \lambda-1}}{\left(\Delta_{r}+2 r \sigma(1-\cos t)\right)^{\lambda+1+s}} d t d r \\
& \leq C(\sin |\theta-\varphi|)^{j} \int_{\frac{1}{2}}^{1}(1-r)^{k} \int_{0}^{\frac{\pi}{2}} \frac{(\sin t)^{2 \lambda-1}}{\left(\Delta_{r}+2 r \sigma(1-\cos t)\right)^{\lambda+1+s}} d t d r \\
& \leq C(\sin |\theta-\varphi|)^{j} \int_{\frac{1}{2}}^{1}(1-r)^{k} \int_{0}^{\frac{\pi}{2}} \frac{t^{2 \lambda-1}}{\left(\Delta_{r}+\sigma t^{2}\right)^{\lambda+1+s}} d t d r \\
& \leq C(\sin |\theta-\varphi|)^{j} \int_{\frac{1}{2}}^{1} \frac{(1-r)^{k}}{\Delta_{r}^{\lambda+s+1}}\left(\sqrt{\frac{\Delta_{r}}{\sigma}}\right)^{2 \lambda} \int_{0}^{\frac{\pi}{2} \sqrt{\frac{\sigma}{\Delta_{r}}}} \frac{u^{2 \lambda-1}}{\left(1+u^{2}\right)^{\lambda+s+1}} d u d r \\
& \leq C \frac{(\sin |\theta-\varphi|)^{j}}{\sigma^{\lambda}} \int_{\frac{1}{2}}^{1} \frac{(1-r)^{k}}{\Delta_{r}^{1+s}} d r \\
& \leq C \frac{(\sin |\theta-\varphi|)^{j}}{\sigma^{\lambda}} \int_{\frac{1}{2}}^{1} \frac{(1-r)^{2 s-j+1 / 2}}{\left(\Delta+(1-r)^{2}\right)^{1+s}} d r \\
& \leq C \frac{(\sin |\theta-\varphi|)^{j}(\sqrt{\Delta})^{2 s-j+3 / 2}}{\sigma^{\lambda} \Delta^{1+s}} \int_{0}^{\frac{1}{2 \sqrt{\Delta}}} \frac{u^{2 s-j+1 / 2}}{\left(1+u^{2}\right)^{1+s}} d u \\
& \leq C \frac{(\sin |\theta-\varphi|)^{j}}{\sigma^{\lambda} \Delta^{\frac{1}{4}+\frac{j}{2}}} \int_{0}^{\infty} \frac{u^{2 s-j+1 / 2}}{\left(1+u^{2}\right)^{1+s}} d u \\
& \leq C \frac{1}{\sigma^{\lambda} \sqrt{|\theta-\varphi|}} \cdot
\end{aligned}
$$


For the second integral we write

$$
\begin{aligned}
I_{2}(\theta, \varphi) & \leq C(\sin \varphi)^{j} \int_{\frac{1}{2}}^{1}(1-r)^{k} \int_{0}^{\frac{\pi}{2}} \frac{t^{2 \lambda+2 j-1}}{\left(\Delta_{r}+\sigma t^{2}\right)^{\lambda+1+s}} d t d r \\
& \leq C(\sin \varphi)^{j} \int_{\frac{1}{2}}^{1}(1-r)^{k} \int_{0}^{\frac{\pi}{2}} \frac{t^{2 \lambda+j-1}}{\left(\Delta_{r}+\sigma t^{2}\right)^{\lambda+1+s}} d t d r \\
& \leq C \frac{(\sin \varphi)^{j}}{\sigma^{\lambda+\frac{j}{2}}} \int_{\frac{1}{2}}^{1} \frac{(1-r)^{k}}{\Delta_{r}^{1+s-\frac{j}{2}}} d r \\
& \leq C \frac{(\sin \varphi)^{j}}{\sigma^{\lambda+\frac{j}{2}}} \int_{\frac{1}{2}}^{1} \frac{(1-r)^{2 s-j+\frac{1}{2}}}{\left(\Delta+(1-r)^{2}\right)^{1+s-\frac{j}{2}}} d r \\
& \leq C \frac{(\sin \varphi)^{j}(\sqrt{\Delta})^{2 s-j+\frac{3}{2}}}{\sigma^{\lambda+\frac{j}{2}} \Delta^{1+s-\frac{j}{2}}} \int_{0}^{\frac{1}{2 \sqrt{\Delta}}} \frac{u^{2 s-j+\frac{1}{2}}}{\left(1+u^{2}\right)^{1+s-\frac{j}{2}}} d u \\
& \leq C \frac{(\sin \varphi)^{j}}{\sigma^{\lambda+\frac{j}{2}} \Delta^{\frac{1}{4}}} \leq C \frac{1}{\sigma^{\lambda} \sqrt{|\theta-\varphi|}},
\end{aligned}
$$

because $\sin \theta \sim \sin \varphi$. Finally it has

$$
\begin{aligned}
I_{3}(\theta, \varphi) & \leq C(\sin \varphi)^{j} \int_{\frac{1}{2}}^{1} \int_{\frac{\pi}{2}}^{\pi}(1-r)^{k} \frac{(\sin t)^{2 \lambda-1}}{\left(\Delta_{r}+\sigma\right)^{\lambda+1+s}} d t d r \\
& \leq C \frac{(\sin \varphi)^{j}}{\sigma^{\lambda+\frac{j}{2}}} \int_{\frac{1}{2}}^{1} \frac{(1-r)^{k}}{\Delta_{r}^{1+s-\frac{j}{2}}} d r \leq C \frac{1}{\sigma^{\lambda} \sqrt{|\theta-\varphi|}} .
\end{aligned}
$$

Then we conclude that if $\frac{\theta}{2} \leq \varphi \leq \frac{3 \theta}{2}, \theta \neq \varphi$,

$$
\left|M_{\ell, s, i, j}(\theta, \varphi)\right| \leq C\left(1+\int_{\frac{1}{2}}^{1} \int_{0}^{\pi}(1-r)^{k} \frac{|b|^{j}(\sin t)^{2 \lambda-1}}{D_{r}^{\lambda+1+s}} d t d r\right) \leq C \frac{1}{\sigma^{\lambda} \sqrt{|\theta-\varphi|}}
$$

that, jointly with (11), gives (9).

We have proved that the integral in (4) is absolutely convergent. By analyzing carefully the above estimates we can also see that, for every $\ell=0,1, \ldots, k-2, R_{\lambda}^{k, \ell}$ is a continuous function on $(0, \pi) \times(0, \pi)$. Then, we conclude $(4)$, for every $\ell=0,1, \ldots, k-1$. Note that when $\ell=k-1$ to prove (4) we need to use distributional arguments (see Lemma 4.2 in Appendix).

Step 2. We now study the kernel

$$
R_{\lambda}^{k}(\theta, \varphi)=\frac{\lambda}{\pi \Gamma(k)} \frac{\partial^{k}}{\partial \theta^{k}}\left[\int_{0}^{1} r^{\lambda-1}\left(\log \frac{1}{r}\right)^{k-1}\left(1-r^{2}\right) \int_{0}^{\pi} \frac{(\sin t)^{2 \lambda-1}}{D_{r}^{\lambda+1}} d t d r\right],
$$

for $\theta, \varphi \in(0, \pi)$. We get estimates which are better than the ones obtained in (5). 
Lemma 2.1. Let $\lambda>0$ and $k \in \mathbb{N}$. Then,

$$
R_{\lambda}^{k}(\theta, \varphi)= \begin{cases}O\left((\sin \varphi)^{-(2 \lambda+1)}\right), & (\theta, \varphi) \in A_{1} \\ \frac{R^{k}(\theta, \varphi)}{(\sin \theta \sin \varphi)^{\lambda}}+O\left(\frac{1}{(\sin \varphi)^{2 \lambda+1}}\left(1+\sqrt{\frac{\sin \varphi}{|\theta-\varphi|}}\right)\right), & (\theta, \varphi) \in A_{2}, \theta \neq \varphi \\ O\left((\sin \theta)^{-(2 \lambda+1)}\right), & (\theta, \varphi) \in A_{3}\end{cases}
$$

where

$$
R^{k}(\theta, \varphi)=\frac{1}{2 \pi \Gamma(k)} \frac{\partial^{k}}{\partial \theta^{k}} \int_{0}^{1}\left(\log \frac{1}{r}\right)^{k-1}\left(\frac{1-r^{2}}{1-2 r \cos (\theta-\varphi)+r^{2}}-1\right) \frac{d r}{r}
$$

Proof. Since $R_{\lambda}^{k}(\theta, \varphi)=(-1)^{k} R_{\lambda}^{k}(\pi-\theta, \pi-\varphi)$ and $R^{k}(\theta, \varphi)=(-1)^{k} R^{k}(\pi-\theta, \pi-\varphi), \theta, \varphi \in$ $(0, \pi)$, we can assume $(\theta, \varphi) \in\left(0, \frac{\pi}{2}\right) \times(0, \pi)$.

When $(\theta, \varphi) \in A_{1} \cup A_{3}$, we can argue as in the proof of (11), for $\ell=k$, and thus we get

$$
\left|R_{\lambda}^{k}(\theta, \varphi)\right|=\left|\frac{\lambda}{\pi \Gamma(k)} \sum_{\substack{s=1, \ldots, k \\ j \geq 2 s-k \\ i+j=s}} c_{k, s, i, j} M_{k, s, i, j}(\theta, \varphi)\right| \leq C \begin{cases}(\sin \varphi)^{-2 \lambda-1}, & \varphi \geq \frac{3 \theta}{2} \\ (\sin \theta)^{-2 \lambda-1}, & \varphi \leq \frac{\theta}{2}\end{cases}
$$

We now consider $\frac{\theta}{2} \leq \varphi \leq \frac{3 \theta}{2}$ and $\varphi \neq \theta$. First we write

$$
\begin{aligned}
R_{\lambda}^{k}(\theta, \varphi)=\frac{\lambda}{\pi \Gamma(k)}[ & \left.\int_{0}^{1} \int_{\frac{\pi}{2}}^{\pi}+\int_{0}^{1-\frac{\sqrt{\sigma}}{2}} \int_{0}^{\frac{\pi}{2}}+\int_{1-\frac{\sqrt{\sigma}}{2}}^{1} \int_{0}^{\frac{\pi}{2}}\right] r^{\lambda-1}\left(\log \frac{1}{r}\right)^{k-1}\left(1-r^{2}\right) \\
& \times \frac{\partial^{k}}{\partial \theta^{k}} \frac{(\sin t)^{2 \lambda-1}}{D_{r}^{\lambda+1}} d t d r \\
= & \frac{\lambda}{\pi \Gamma(k)}\left[I_{\lambda}^{k, 1}(\theta, \varphi)+I_{\lambda}^{k, 2}(\theta, \varphi)+I_{\lambda}^{k, 3}(\theta, \varphi)\right] .
\end{aligned}
$$

Let us decompose $I_{\lambda}^{k, 3}(\theta, \varphi)$ as follows,

$$
\begin{aligned}
I_{\lambda}^{k, 3}(\theta, \varphi) & =\int_{1-\frac{\sqrt{\sigma}}{2}}^{1} r^{\lambda-1}\left(\log \frac{1}{r}\right)^{k-1}\left(1-r^{2}\right) \frac{\partial^{k}}{\partial \theta^{k}} \int_{0}^{\frac{\pi}{2}}\left(\frac{(\sin t)^{2 \lambda-1}}{D_{r}^{\lambda+1}}-\frac{t^{2 \lambda-1}}{\left(\Delta_{r}+r \sigma t^{2}\right)^{\lambda+1}}\right) d t d r \\
& +\int_{1-\frac{\sqrt{\sigma}}{2}}^{1} r^{\lambda-1}\left(\log \frac{1}{r}\right)^{k-1}\left(1-r^{2}\right) \frac{\partial^{k}}{\partial \theta^{k}} \int_{0}^{\frac{\pi}{2}} \frac{t^{2 \lambda-1}}{\left(\Delta_{r}+r \sigma t^{2}\right)^{\lambda+1}} d t d r \\
(13) & =J_{\lambda}^{k}(\theta, \varphi)+K_{\lambda}^{k}(\theta, \varphi) .
\end{aligned}
$$


Moreover, we observe that by making the change of variable $u=\sqrt{\frac{r \sigma}{\Delta_{r}}} t$,

$$
\begin{aligned}
\int_{0}^{\frac{\pi}{2}} \frac{t^{2 \lambda-1}}{\left(\Delta_{r}+r \sigma t^{2}\right)^{\lambda+1}} d t & =\left(\int_{0}^{\infty}-\int_{\frac{\pi}{2}}^{\infty}\right) \frac{t^{2 \lambda-1}}{\left(\Delta_{r}+r \sigma t^{2}\right)^{\lambda+1}} d t \\
& =\frac{1}{(r \sigma)^{\lambda} \Delta_{r}} \int_{0}^{\infty} \frac{u^{2 \lambda-1}}{\left(1+u^{2}\right)^{\lambda+1}} d u-\int_{\frac{\pi}{2}}^{\infty} \frac{t^{2 \lambda-1}}{\left(\Delta_{r}+r \sigma t^{2}\right)^{\lambda+1}} d t \\
& =\frac{1}{2 \lambda(r \sigma)^{\lambda} \Delta_{r}}-\int_{\frac{\pi}{2}}^{\infty} \frac{t^{2 \lambda-1}}{\left(\Delta_{r}+r \sigma t^{2}\right)^{\lambda+1}} d t, \quad r \in(0,1) .
\end{aligned}
$$

Then, Leibniz's rule leads to

$$
\begin{aligned}
K_{\lambda}^{k}(\theta, \varphi) & =\frac{1}{2 \lambda}\left[\int_{1-\frac{\sqrt{\sigma}}{2}}^{1}\left(\log \frac{1}{r}\right)^{k-1} \frac{\partial^{k}}{\partial \theta^{k}}\left(\frac{1}{\sigma^{\lambda}} \frac{\left(1-r^{2}\right)}{\Delta_{r}}\right) \frac{d r}{r}\right] \\
& -\int_{1-\frac{\sqrt{\sigma}}{2}}^{1} r^{\lambda-1}\left(\log \frac{1}{r}\right)^{k-1}\left(1-r^{2}\right) \frac{\partial^{k}}{\partial \theta^{k}} \int_{\frac{\pi}{2}}^{\infty} \frac{t^{2 \lambda-1}}{\left(\Delta_{r}+r \sigma t^{2}\right)^{\lambda+1}} d t d r \\
& =\frac{1}{2 \lambda \sigma^{\lambda}}\left[\int_{1-\frac{\sqrt{\sigma}}{2}}^{1}\left(\log \frac{1}{r}\right)^{k-1} \frac{\partial^{k}}{\partial \theta^{k}} \frac{\left(1-r^{2}\right)}{\Delta_{r}} \frac{d r}{r}\right] \\
& +\frac{1}{2 \lambda} \sum_{n=0}^{k-1}\left(\begin{array}{c}
k \\
n
\end{array}\right) \frac{\partial^{k-n}}{\partial \theta^{k-n}}\left(\frac{1}{\sigma^{\lambda}}\right)\left[\int_{1-\frac{\sqrt{\sigma}}{2}}^{1}\left(\log \frac{1}{r}\right)^{k-1} \frac{\partial^{n}}{\partial \theta^{n}} \frac{\left(1-r^{2}\right)}{\Delta_{r}} \frac{d r}{r}\right] \\
& -\int_{1-\frac{\sqrt{\sigma}}{2}}^{1} r^{\lambda-1}\left(\log \frac{1}{r}\right)^{k-1}\left(1-r^{2}\right) \frac{\partial^{k}}{\partial \theta^{k}} \int_{\frac{\pi}{2}}^{\infty} \frac{t^{2 \lambda-1}}{\left(\Delta_{r}+r \sigma t^{2}\right)^{\lambda+1}} d t d r .
\end{aligned}
$$

We observe that

$$
K_{\lambda}^{k}(\theta, \varphi)=\frac{\pi \Gamma(k)}{\lambda \sigma^{\lambda}} R^{k}(\theta, \varphi)+\sum_{\beta=1}^{3} K_{\lambda}^{k, \beta}(\theta, \varphi)
$$

where

$$
\begin{aligned}
K_{\lambda}^{k, 1}(\theta, \varphi) & =-\frac{1}{2 \lambda \sigma^{\lambda}}\left[\int_{0}^{1-\frac{\sqrt{\sigma}}{2}}\left(\log \frac{1}{r}\right)^{k-1} \frac{\partial^{k}}{\partial \theta^{k}} \frac{\left(1-r^{2}\right)}{\Delta_{r}} \frac{d r}{r}\right] \\
K_{\lambda}^{k, 2}(\theta, \varphi) & =\frac{1}{2 \lambda} \sum_{n=0}^{k-1}\left(\begin{array}{l}
k \\
n
\end{array}\right) \frac{\partial^{k-n}}{\partial \theta^{k-n}}\left(\frac{1}{\sigma^{\lambda}}\right)\left[\int_{1-\frac{\sqrt{\sigma}}{2}}^{1}\left(\log \frac{1}{r}\right)^{k-1} \frac{\partial^{n}}{\partial \theta^{n}} \frac{\left(1-r^{2}\right)}{\Delta_{r}} \frac{d r}{r}\right],
\end{aligned}
$$

and

$$
K_{\lambda}^{k, 3}(\theta, \varphi)=-\int_{1-\frac{\sqrt{\sigma}}{2}}^{1} r^{\lambda-1}\left(\log \frac{1}{r}\right)^{k-1}\left(1-r^{2}\right) \frac{\partial^{k}}{\partial \theta^{k}} \int_{\frac{\pi}{2}}^{\infty} \frac{t^{2 \lambda-1}}{\left(\Delta_{r}+r \sigma t^{2}\right)^{\lambda+1}} d t d r .
$$

Thus, according to (12), (13) and (14), to establish our result we must analyze $I_{\lambda}^{k, \beta}(\theta, \varphi)$, $\beta=1,2, J_{\lambda}^{k}(\theta, \varphi)$ and $K_{\lambda}^{k, \beta}(\theta, \varphi), \beta=1,2,3$. 
Let us consider first $I_{\lambda}^{k, 1}(\theta, \varphi)$. We will see that

$$
\left|I_{\lambda}^{k, 1}(\theta, \varphi)\right| \leq \frac{C}{(\sin \varphi)^{2 \lambda+1}}\left(1+\sqrt{\frac{\sin \varphi}{|\theta-\varphi|}}\right) .
$$

Let $s=1, \ldots, k, j \geq 2 s-k$ and $i+j=s$. We define

$$
I_{\lambda, s, i, j}^{k, 1}(\theta, \varphi)=\int_{0}^{1} r^{i+j+\lambda-1}\left(\log \frac{1}{r}\right)^{k-1}\left(1-r^{2}\right) \int_{\frac{\pi}{2}}^{\pi} \frac{a^{i} b^{j}(\sin t)^{2 \lambda-1}}{D_{r}^{\lambda+s+1}} d t d r .
$$

According to (6) it is then sufficient to obtain (15) when $I_{\lambda, s, i, j}^{k, 1}(\theta, \varphi)$ replaces to $I_{\lambda}^{k, 1}(\theta, \varphi)$. By proceeding as in Step 1, using (10), since $D_{r} \geq C$, for $0<r<\frac{1}{2}$, and $D_{r} \geq\left(\Delta_{r}+\sigma\right)$, for $\frac{1}{2}<r<1$ and $t \in\left(\frac{\pi}{2}, \pi\right)$, it has

$$
\begin{aligned}
\left|I_{\lambda, s, i, j}^{k, 1}(\theta, \varphi)\right| & \leq C \int_{0}^{1} r^{\lambda-1}\left(\log \frac{1}{r}\right)^{k-1}\left(1-r^{2}\right) \int_{\frac{\pi}{2}}^{\pi} \frac{|b|^{j}(\sin t)^{2 \lambda-1}}{D_{r}^{\lambda+s+1}} d t d r \\
& \leq C\left(1+(\sin \varphi)^{j} \int_{\frac{1}{2}}^{1} \frac{(1-r)^{k}}{\left(\Delta_{r}+\sigma\right)^{\lambda+s+1}} d r\right) \\
& \leq C\left(1+\frac{(\sin \varphi)^{j}}{\sigma^{\lambda+\frac{1}{4}+\frac{j}{2}}} \int_{\frac{1}{2}}^{1} \frac{(1-r)^{2 s-j}}{\Delta_{r}^{s+\frac{3}{4}-\frac{j}{2}}} d r\right) \\
& \leq C\left(1+\frac{1}{\sigma^{\lambda+\frac{1}{4}} \Delta^{\frac{1}{4}}} \int_{0}^{\infty} \frac{u^{2 s-j}}{\left(1+u^{2}\right)^{s+\frac{3}{4}-\frac{j}{2}}} d u\right) \\
& \leq C\left(1+\frac{1}{(\sin \varphi)^{2 \lambda+\frac{1}{2}} \sqrt{|\theta-\varphi|}}\right) \leq \frac{C}{(\sin \varphi)^{2 \lambda+1}}\left(1+\sqrt{\frac{\sin \varphi}{|\theta-\varphi|}}\right)
\end{aligned}
$$

For $I_{\lambda}^{k, 2}(\theta, \varphi)$ we proceed in a similar way. Consider $s=1, \ldots, k, j \geq 2 s-k$, and $i+j=s$, and

$$
I_{\lambda, s, i, j}^{k, 2}(\theta, \varphi)=\int_{0}^{1-\frac{\sqrt{\sigma}}{2}} r^{i+j+\lambda-1}\left(\log \frac{1}{r}\right)^{k-1}\left(1-r^{2}\right) \int_{0}^{\frac{\pi}{2}} \frac{a^{i} b^{j}(\sin t)^{2 \lambda-1}}{D_{r}^{\lambda+s+1}} d t d r .
$$

We have that

$$
\begin{aligned}
\left|I_{\lambda, s, i, j}^{k, 2}(\theta, \varphi)\right| & \leq C\left(\int_{0}^{\frac{1}{2}} r^{\lambda-1}\left(\log \frac{1}{r}\right)^{k-1} d r+(\sin \varphi)^{j} \int_{\frac{1}{2}}^{1-\frac{\sqrt{\sigma}}{2}} \frac{(1-r)^{k}}{\Delta_{r}^{\lambda+s+1}} d r\right) \\
& \leq C\left(1+(\sin \varphi)^{j} \int_{\frac{1}{2}}^{1-\frac{\sqrt{\sigma}}{2}} \frac{(1-r)^{2 s-j}}{(1-r)^{2 \lambda+2 s+2}} d r\right) \leq \frac{C}{(\sin \varphi)^{2 \lambda+1}} .
\end{aligned}
$$

Hence,

$$
\left|I_{\lambda}^{k, 2}(\theta, \varphi)\right| \leq \frac{C}{(\sin \varphi)^{2 \lambda+1}}
$$


To estimate $J_{\lambda}^{k}(\theta, \varphi)$ (see $\left.(13)\right)$, we write

$$
J_{\lambda}^{k}(\theta, \varphi)=J_{\lambda}^{k, 1}(\theta, \varphi)+J_{\lambda}^{k, 2}(\theta, \varphi)
$$

where

$$
J_{\lambda}^{k, 1}(\theta, \varphi)=\int_{1-\frac{\sqrt{\sigma}}{2}}^{1} r^{\lambda-1}\left(\log \frac{1}{r}\right)^{k-1}\left(1-r^{2}\right) \frac{\partial^{k}}{\partial \theta^{k}} \int_{0}^{\frac{\pi}{2}} \frac{(\sin t)^{2 \lambda-1}-t^{2 \lambda-1}}{D_{r}^{\lambda+1}} d t d r
$$

and

$$
J_{\lambda}^{k, 2}(\theta, \varphi)=\int_{1-\frac{\sqrt{\sigma}}{2}}^{1} r^{\lambda-1}\left(\log \frac{1}{r}\right)^{k-1}\left(1-r^{2}\right) \frac{\partial^{k}}{\partial \theta^{k}} \int_{0}^{\frac{\pi}{2}} t^{2 \lambda-1}\left(\frac{1}{D_{r}^{\lambda+1}}-\frac{1}{\left(\Delta_{r}+r \sigma t^{2}\right)^{\lambda+1}}\right) d t d r
$$

To analyze $J_{\lambda}^{k, 1}(\theta, \varphi)$ assume, as above, $s=1, \ldots, k, j \geq 2 s-k$, and $i+j=s$ and consider

$$
J_{\lambda, s, i, j}^{k, 1}(\theta, \varphi)=\int_{1-\frac{\sqrt{\sigma}}{2}}^{1} r^{i+j+\lambda-1}\left(\log \frac{1}{r}\right)^{k-1}\left(1-r^{2}\right) \int_{0}^{\frac{\pi}{2}} \frac{a^{i} b^{j}\left[(\sin t)^{2 \lambda-1}-t^{2 \lambda-1}\right]}{D_{r}^{\lambda+s+1}} d t d r .
$$

By using the mean value theorem and that $|b|^{j} \leq C\left(|\theta-\varphi|^{j}+\left(t^{2} \sin \varphi\right)^{j}\right), t \in\left(0, \frac{\pi}{2}\right)$, we have

$$
\begin{aligned}
\left|J_{\lambda, s, i, j}^{k, 1}(\theta, \varphi)\right| & \leq C \int_{1-\frac{\sqrt{\sigma}}{2}}^{1}(1-r)^{k} \int_{0}^{\frac{\pi}{2}} \frac{|b|^{j} t^{2 \lambda+1}}{D_{r}^{\lambda+s+1}} d t d r \\
& \leq C \int_{1-\frac{\sqrt{\sigma}}{2}}^{1}(1-r)^{k} \int_{0}^{\frac{\pi}{2}} \frac{\left[|\theta-\varphi|^{j}+\left(t^{2} \sin \varphi\right)^{j}\right] t^{2 \lambda+1}}{\left(\Delta_{r}+\sigma t^{2}\right)^{\lambda+s+1}} d t d r \\
& =C\left(J_{\lambda, s, i, j}^{k, 1,1}(\theta, \varphi)+J_{\lambda, s, i, j}^{k, 1,2}(\theta, \varphi)\right) .
\end{aligned}
$$

We can obtain for each term in the last sum the following estimates. Firstly,

$$
\begin{aligned}
\left|J_{\lambda, s, i, j}^{k, 1,1}(\theta, \varphi)\right| & \leq C \frac{|\theta-\varphi|^{j}}{\sigma^{\lambda+1}} \int_{1-\frac{\sqrt{\sigma}}{2}}^{1} \frac{(1-r)^{k}}{\Delta_{r}^{s}} \int_{0}^{\frac{\pi}{2} \sqrt{\frac{\sigma}{\Delta_{r}}}} \frac{u^{2 \lambda+1}}{\left(1+u^{2}\right)^{\lambda+s+1}} d u d r \\
& \leq C \frac{|\theta-\varphi|^{j}}{\sigma^{\lambda+1}} \int_{1-\frac{\sqrt{\sigma}}{2}}^{1} \frac{(1-r)^{k}}{\left(\Delta+(1-r)^{2}\right)^{s-\frac{j}{2}+\frac{j}{2}}} d r \\
& \leq C \frac{1}{\sigma^{\lambda+1}} \int_{1-\frac{\sqrt{\sigma}}{2}}^{1}(1-r)^{k-2 s+j} d r \leq C \frac{\sigma^{\frac{k+j}{2}-s}}{\sigma^{\lambda+\frac{1}{2}}} \leq \frac{C}{(\sin \varphi)^{2 \lambda+1}}
\end{aligned}
$$


We also have

$$
\begin{aligned}
\left|J_{\lambda, s, i, j}^{k, 1,2}(\theta, \varphi)\right| & \leq C(\sin \varphi)^{j} \int_{1-\frac{\sqrt{\sigma}}{2}}^{1}(1-r)^{k} \int_{0}^{\frac{\pi}{2}} \frac{t^{2 \lambda+2 j+1}}{\left((1-r)^{2}+\sigma t^{2}\right)^{\lambda+s+1}} d t d r \\
& \leq C \frac{(\sin \varphi)^{j}}{\sigma^{\lambda+\frac{j}{2}+\frac{3}{4}}} \int_{1-\frac{\sqrt{\sigma}}{2}}^{1}(1-r)^{k-2 s+j-\frac{1}{2}} d r \int_{0}^{\frac{\pi}{2}} t^{j-\frac{1}{2}} d t \\
& \leq C \frac{\sigma^{\frac{k+j}{2}-s}}{\sigma^{\lambda+\frac{1}{2}}} \leq \frac{C}{(\sin \varphi)^{2 \lambda+1}} .
\end{aligned}
$$

Thus we get by (6)

$$
\left|J_{\lambda}^{k, 1}(\theta, \varphi)\right| \leq \frac{C}{(\sin \varphi)^{2 \lambda+1}}
$$

In the same way, and according to $(6)$, to see the estimate for $J_{\lambda}^{k, 2}(\theta, \varphi)$ we analyze

$$
\begin{aligned}
J_{\lambda, s, i, j}^{k, 2}(\theta, \varphi) & =\int_{1-\frac{\sqrt{\sigma}}{2}}^{1} r^{\lambda+i+j-1}\left(\log \frac{1}{r}\right)^{k-1}\left(1-r^{2}\right) \\
& \times \int_{0}^{\frac{\pi}{2}} t^{2 \lambda-1}\left(\frac{a^{i} b^{j}}{D_{r}^{\lambda+s+1}}-\frac{A^{i} B^{j}}{\left(\Delta_{r}+r \sigma t^{2}\right)^{\lambda+s+1}}\right) d t d r
\end{aligned}
$$

for every $s=1, \ldots, k, j \geq 2 s-k$, and $i+j=s$. Here $A=\cos (\theta-\varphi)-\frac{\sigma t^{2}}{2}$ and $B=\frac{\partial A}{\partial \theta}$.

By using the mean value theorem we obtain, for $t \in\left(0, \frac{\pi}{2}\right)$,

$$
\begin{aligned}
\mid \frac{a^{i} b^{j}}{D_{r}^{\lambda+s+1}} & -\frac{A^{i} B^{j}}{\left(\Delta_{r}+r \sigma t^{2}\right)^{\lambda+s+1}} \mid \\
& \leq\left|a^{i} b^{j}\left(\frac{1}{D_{r}^{\lambda+s+1}}-\frac{1}{\left(\Delta_{r}+r \sigma t^{2}\right)^{\lambda+s+1}}\right)\right|+\left|\frac{\left(a^{i}-A^{i}\right) b^{j}+A^{i}\left(b^{j}-B^{j}\right)}{\left(\Delta_{r}+r \sigma t^{2}\right)^{\lambda+s+1}}\right| \\
& \leq C\left(\frac{|b|^{j} \sigma t^{4}}{\left(\Delta_{r}+r \sigma t^{2}\right)^{\lambda+s+2}}+\frac{|b|^{j-1} \sqrt{\sigma} t^{4}}{\left(\Delta_{r}+r \sigma t^{2}\right)^{\lambda+s+1}}\right) \\
& \leq C\left(\frac{|b|^{j} t^{2}}{\left(\Delta_{r}+\sigma t^{2}\right)^{\lambda+s+1}}+\frac{|b|^{j-1} \sqrt{\sigma} t^{3}}{\left(\Delta_{r}+\sigma t^{2}\right)^{\lambda+s+1}}\right),
\end{aligned}
$$

where the second term in the two last sums does not appear when $j=0$. Then, we write

$$
\left|J_{\lambda, s, i, j}^{k, 2}(\theta, \varphi)\right| \leq C\left(J_{\lambda, s, i, j}^{k, 2,1}(\theta, \varphi)+J_{\lambda, s, i, j}^{k, 2,2}(\theta, \varphi)\right),
$$

where

$$
J_{\lambda, s, i, j}^{k, 2,1}(\theta, \varphi)=\int_{1-\frac{\sqrt{\sigma}}{2}}^{1}(1-r)^{k} \int_{0}^{\frac{\pi}{2}} \frac{|b|^{j} t^{2 \lambda+1}}{\left(\Delta_{r}+\sigma t^{2}\right)^{\lambda+s+1}} d t d r
$$

and

$$
J_{\lambda, s, i, j}^{k, 2,2}(\theta, \varphi)=\sqrt{\sigma} \int_{1-\frac{\sqrt{\sigma}}{2}}^{1}(1-r)^{k} \int_{0}^{\frac{\pi}{2}} \frac{|b|^{j-1} t^{2 \lambda+2}}{\left(\Delta_{r}+\sigma t^{2}\right)^{\lambda+s+1}} d t d r, \quad \text { when } j \geq 1
$$


We observe that $J_{\lambda, s, i, j}^{k, 2,1}(\theta, \varphi)$ was already analyzed in (17). On the other hand, when $j \geq 1$, we can use that $|b|^{j-1} \leq C\left(|\theta-\varphi|^{j-1}+\left(t^{2} \sin \varphi\right)^{j-1}\right), t \in\left(0, \frac{\pi}{2}\right)$, and proceed as in the estimation of (17) to study $J_{\lambda, s, i, j}^{k, 2,2}$. Thus we get that

$$
\left|J_{\lambda}^{k, 2}(\theta, \varphi)\right| \leq \frac{C}{(\sin \varphi)^{2 \lambda+1}}
$$

By combining (18) and (19) we conclude that

$$
\left|J_{\lambda}^{k}(\theta, \varphi)\right| \leq \frac{C}{(\sin \varphi)^{2 \lambda+1}}
$$

Finally we deal with $K_{\lambda}^{k, \beta}(\theta, \varphi), \beta=1,2,3$ (see (14)).

By invoking (6) with $t=0$ and $\lambda=0$, it has that

$$
\frac{\partial^{\ell}}{\partial \theta^{\ell}}\left(\frac{1}{\Delta_{r}}\right)=\sum_{s, i, j} c_{\ell, s, i, j} \frac{r^{i+j}(\cos (\theta-\varphi))^{i}(-\sin (\theta-\varphi))^{j}}{\Delta_{r}^{1+s}}, \quad \ell \in \mathbb{N},
$$

where $c_{\ell, s, i, j} \neq 0$ only if $s=1, \ldots, \ell, j \geq 2 s-\ell$ and $i+j=s$.

Also, for every $n=0, \ldots, k-1$,

$$
\left|\frac{\partial^{k-n}}{\partial \theta^{k-n}}\left(\frac{1}{\sigma^{\lambda}}\right)\right| \leq C(\sin \theta)^{-\lambda-k+n}(\sin \varphi)^{-\lambda} \leq C \sigma^{-\lambda-\frac{k-n}{2}}
$$

Hence, by proceeding as above, to estimate $K_{\lambda}^{k, \beta}(\theta, \varphi), \beta=1,2,3$, it is sufficient to study the following integrals:

$$
K_{\lambda, s, i, j}^{k, 1}(\theta, \varphi)=\frac{1}{\sigma^{\lambda}} \int_{0}^{1-\frac{\sqrt{\sigma}}{2}} r^{i+j-1}\left(\log \frac{1}{r}\right)^{k-1} \frac{\left(1-r^{2}\right)(\cos (\theta-\varphi))^{i}(-\sin (\theta-\varphi))^{j}}{\Delta_{r}^{s+1}} d r
$$

when $s=1, \ldots, k, j \geq 2 s-k$ and $i+j=s$;

$$
\begin{gathered}
K_{\lambda}^{k, 2,0}(\theta, \varphi)=\sigma^{-k / 2-\lambda} \int_{1-\frac{\sqrt{\sigma}}{2}}^{1}\left(\log \frac{1}{r}\right)^{k-1} \frac{\left(1-r^{2}\right)}{\Delta_{r}} \frac{d r}{r} \\
K_{\lambda, s, i, j}^{k, 2, n}(\theta, \varphi)=\frac{\partial^{k-n}}{\partial \theta^{k-n}}\left(\frac{1}{\sigma^{\lambda}}\right) \int_{1-\frac{\sqrt{\sigma}}{2}}^{1} r^{i+j-1}\left(\log \frac{1}{r}\right)^{k-1} \frac{\left(1-r^{2}\right)(\cos (\theta-\varphi))^{i}(-\sin (\theta-\varphi))^{j}}{\Delta_{r}^{s+1}} d r,
\end{gathered}
$$

for each $n=1, \ldots, k-1, s=1, \ldots, n, j \geq 2 s-n$, and $i+j=s$; and

$$
K_{\lambda, s, i, j}^{k, 3}(\theta, \varphi)=\int_{1-\frac{\sqrt{\sigma}}{2}}^{1} r^{i+j+\lambda-1}\left(\log \frac{1}{r}\right)^{k-1}\left(1-r^{2}\right) \int_{\frac{\pi}{2}}^{\infty} \frac{A^{i} B^{j} t^{2 \lambda-1}}{\left(\Delta_{r}+r \sigma t^{2}\right)^{\lambda+s+1}} d t d r,
$$

when $s=1, \ldots, k, j \geq 2 s-k$, and $i+j=s$. Here, as before, $A=\cos (\theta-\varphi)-\frac{\sigma t^{2}}{2}$ and $B=\frac{\partial A}{\partial \theta}$. 
Consider $s=1, \ldots, k, j \geq 2 s-k$, and $i+j=s$. We can write

$$
\begin{aligned}
\left|K_{\lambda, s, i, j}^{k, 1}(\theta, \varphi)\right| & \leq C \frac{1}{\sigma^{\lambda}}\left(\int_{0}^{\frac{1}{2}} r^{i+j-1}\left(\log \frac{1}{r}\right)^{k-1} d r+\int_{\frac{1}{2}}^{1-\frac{\sqrt{\sigma}}{2}} \frac{(1-r)^{k}|\theta-\varphi|^{j}}{\left(\Delta+(1-r)^{2}\right)^{s+1}} d r\right) \\
& \leq C \frac{1}{\sigma^{\lambda}}\left(1+\frac{|\theta-\varphi|^{j}}{\Delta^{\frac{j}{2}}} \int_{\frac{1}{2}}^{1-\frac{\sqrt{\sigma}}{2}}(1-r)^{k-2 s+j-2} d r\right) \\
& \leq C \frac{1}{\sigma^{\lambda}}\left(1+\frac{\sigma^{\frac{k+j}{2}-s}}{\sqrt{\sigma}}\right) \leq \frac{C}{(\sin \varphi)^{2 \lambda+1}} .
\end{aligned}
$$

On the other hand, by using (21), for each $n=1, \ldots, k-1, s=1, \ldots, n, j \geq 2 s-n$, and $i+j=s$, we obtain

$$
\begin{aligned}
\left|K_{\lambda, s, i, j}^{k, 2, n}(\theta, \varphi)\right| & \leq C \frac{|\theta-\varphi|^{j}}{\sigma^{\lambda+\frac{k-n}{2}}} \int_{1-\frac{\sqrt{\sigma}}{2}}^{1} \frac{(1-r)^{k}}{\left(\Delta+(1-r)^{2}\right)^{s+1}} d r \\
& \leq C \frac{|\theta-\varphi|^{j}}{\sigma^{\lambda+\frac{k-n}{2}} \Delta^{\frac{j}{2}+\frac{1}{4}}} \int_{1-\frac{\sqrt{\sigma}}{2}}^{1}(1-r)^{k-2 s+j-\frac{3}{2}} d r \\
& \leq C \frac{\sigma^{\frac{n+j}{2}-s}}{\sigma^{\lambda+\frac{1}{4}} \Delta^{\frac{1}{4}}} \leq \frac{C}{(\sin \varphi)^{2 \lambda+1}} \sqrt{\frac{\sin \varphi}{|\theta-\varphi|}}
\end{aligned}
$$

In a similar way we obtain

$$
\left|K_{\lambda}^{k, 2,0}(\theta, \varphi)\right| \leq \frac{C}{(\sin \varphi)^{2 \lambda+1}} \sqrt{\frac{\sin \varphi}{|\theta-\varphi|}} .
$$

Finally, assume $s=1, \ldots, k, j \geq 2 s-k$, and $i+j=s$. By taking into account that $|B|^{j} \leq C\left(t^{2} \sin \varphi\right)^{j}$ and $|A|^{i} \leq C\left(1+\sigma^{i} t^{2 i}\right), t \geq \frac{\pi}{2}$, and the last formula in [10, p. 37], we obtain

$$
\begin{aligned}
K_{\lambda, s, i, j}^{k, 3}(\theta, \varphi) & \leq C \int_{1-\frac{\sqrt{\sigma}}{2}}^{1}(1-r)^{k} \int_{\frac{\pi}{2}}^{\infty} \frac{|A|^{i}|B|^{j} t^{2 \lambda-1}}{\left(\Delta_{r}+\sigma t^{2}\right)^{\lambda+s+1}} d t d r \\
& \leq C \frac{(\sin \varphi)^{j}}{\sigma^{\lambda+j}}\left(\int_{1-\frac{\sqrt{\sigma}}{2}}^{1} \frac{(1-r)^{k}}{\Delta_{r}^{s+1-j}} \int_{\frac{\pi}{2} \sqrt{\frac{\sigma}{\Delta_{r}}}}^{\infty} \frac{u^{2 \lambda+2 j-1}}{\left(1+u^{2}\right)^{\lambda+s+1}} d u d r\right. \\
& \left.+\int_{1-\frac{\sqrt{\sigma}}{2}}^{1} \frac{(1-r)^{k}}{\Delta_{r}} \int_{\frac{\pi}{2} \sqrt{\frac{\sigma}{\Delta_{r}}}}^{\infty} \frac{u^{2 \lambda+2 j+2 i-1}}{\left(1+u^{2}\right)^{\lambda+s+1}} d u d r\right) \\
& \leq C \frac{(\sin \varphi)^{j}}{\sigma^{\lambda+j+1}}\left(\int_{1-\frac{\sqrt{\sigma}}{2}}^{1} \frac{(1-r)^{k}}{\left(\Delta+(1-r)^{2}\right)^{s-j}} d r+\int_{1-\frac{\sqrt{\sigma}}{2}}^{1}(1-r)^{k} d r\right) \\
& \leq C \frac{(\sin \varphi)^{j}}{\sigma^{\lambda+j+1}} \int_{1-\frac{\sqrt{\sigma}}{2}}^{1}(1-r)^{k-2 s+2 j} d r \leq C \frac{(\sin \varphi)^{j} \sigma^{\frac{k+j}{2}-s}}{\sigma^{\lambda+\frac{j}{2}+\frac{1}{2}}} \leq \frac{C}{(\sin \varphi)^{2 \lambda+1}}
\end{aligned}
$$


Thus, we have obtained that

$$
\sum_{\beta=1}^{3}\left|K_{\lambda}^{k, \beta}(\theta, \varphi)\right| \leq \frac{C}{(\sin \varphi)^{2 \lambda+1}}\left(1+\sqrt{\frac{\sin \varphi}{|\theta-\varphi|}}\right) .
$$

By considering (12), (13), (14) and the estimations (15), (16), (20) and (22) we conclude that, when $\frac{\theta}{2} \leq \varphi \leq \frac{3 \theta}{2}, \theta \neq \varphi$,

$$
\left|R_{\lambda}^{k}(\theta, \varphi)-\frac{R^{k}(\theta, \varphi)}{\sigma^{\lambda}}\right| \leq \frac{C}{(\sin \varphi)^{2 \lambda+1}}\left(1+\sqrt{\frac{\sin \varphi}{|\theta-\varphi|}}\right),
$$

and the proof of Lemma 2.1 is finished.

Step 3. We now establish that the $k$-th Riesz transform in the circle is a principal value integral operator, that is,

$$
\begin{aligned}
\frac{d^{k}}{d \theta^{k}} \int_{0}^{\pi} f(\varphi) \int_{0}^{1} & \left(\log \frac{1}{r}\right)^{k-1}\left(\frac{1-r^{2}}{1-2 r \cos (\theta-\varphi)+r^{2}}-1\right) \frac{d r}{r} d \varphi \\
& =2 \pi \Gamma(k) \lim _{\varepsilon \rightarrow 0^{+}} \int_{0,|\theta-\varphi|>\varepsilon}^{\pi} f(\varphi) R^{k}(\theta, \varphi) d \varphi+\beta_{k} f(\theta)
\end{aligned}
$$

for every $\theta \in(0, \pi)$, and where $\beta_{k}=0$ when $k$ is odd, and $\beta_{k}=2 \pi(-1)^{\frac{k}{2}} \Gamma(k)$, when $k$ is even.

Let us consider the function

$$
H^{k}(\omega)=\int_{0}^{1}\left(\log \frac{1}{r}\right)^{k-1} \frac{\partial^{k-1}}{\partial \omega^{k-1}}\left(\frac{1-r^{2}}{1-2 r \cos \omega+r^{2}}-1\right) \frac{d r}{r}, \quad \omega \in \mathbb{R} \backslash\{2 k \pi: k \in \mathbb{Z}\} .
$$

Firstly we are going to analyze the behavior of $H^{k}(w)$ when $w \rightarrow 0^{+}$. We have that

$$
\begin{aligned}
H^{1}(w) & =\int_{0}^{1}\left(\frac{1-r^{2}}{1-2 r \cos w+r^{2}}-1\right) \frac{d r}{r} \\
& =-\log (2(1-\cos w)), \quad w \in \mathbb{R} \backslash\{2 k \pi: k \in \mathbb{Z}\} .
\end{aligned}
$$

Then, $\lim _{w \rightarrow 0} w H^{1}(w)=0$.

Assume that $k \in \mathbb{N}, k \geq 2$. According to (6), it has

$$
\begin{aligned}
H^{k}(w) & =\sum_{\substack{s=1, \ldots, k-1 \\
j \geq 2 s-k+1 \\
i+j=s}} c_{k-1, s, i, j}\left(\int_{0}^{\frac{1}{2}}+\int_{\frac{1}{2}}^{1}\right) \frac{r^{i+j}(\cos w)^{i}(-\sin w)^{j}}{\left(1-2 r \cos w+r^{2}\right)^{s+1}}\left(1-r^{2}\right)\left(\log \frac{1}{r}\right)^{k-1} \frac{d r}{r} \\
& =\sum_{\substack{s=1, \ldots, k-1 \\
j \geq 2 s-k+1 \\
i+j=s}} c_{k-1, s, i, j}\left(I_{k, s, i, j}^{0}(w)+I_{k, s, i, j}^{1}(w)\right), \quad w \in \mathbb{R} \backslash\{2 k \pi: k \in \mathbb{Z}\} .
\end{aligned}
$$


Let $s=1, \ldots, k-1, i+j=s, j \geq 2 s-k+1$. By using the dominated convergence theorem we obtain

$$
\lim _{w \rightarrow 0} I_{k, s, i, j}^{0}(w)= \begin{cases}0, & j \geq 1 \\ \int_{0}^{1 / 2}\left(\log \frac{1}{r}\right)^{k-1} \frac{\left(1-r^{2}\right) r^{s-1}}{(1-r)^{2(s+1)}} d r, & j=0\end{cases}
$$

and, when $2 s-k+1<0$,

$$
\lim _{w \rightarrow 0} I_{k, s, i, j}^{1}(w)= \begin{cases}0, & j \geq 1 \\ \int_{1 / 2}^{1}\left(\log \frac{1}{r}\right)^{k-1} \frac{\left(1-r^{2}\right) r^{s-1}}{(1-r)^{2(s+1)}} d r, & j=0 .\end{cases}
$$

Assume now $2 s-k+1 \geq 0$. We have that

$$
\left|I_{k, s, i, j}^{1}(w)\right| \leq C|w|^{j+k-2 s-1} \int_{0}^{\frac{1}{2|w|}} \frac{u^{k}}{\left(1+u^{2}\right)^{s+1}} d u \leq C|w|^{j+k-2 s-\frac{3}{2}}, \quad w \in(-\pi, \pi) \backslash\{0\} .
$$

Then,

$$
\lim _{w \rightarrow 0} w I_{k, s, i, j}^{1}(w)=0
$$

and if $j>2 s-k+1$,

$$
\lim _{w \rightarrow 0} I_{k, s, i, j}^{1}(w)=0 .
$$

Also, if $j=2 s-k+1>0$ (as will be the case if $\mathrm{k}$ is even), by using mean value theorem it follows

$$
\begin{aligned}
\lim _{w \rightarrow 0^{+}} I_{k, s, i, j}^{1}(w) & =-2 \lim _{w \rightarrow 0^{+}}\left(\frac{\sin w}{w}\right)^{2 s-k+1} \int_{0}^{\frac{1}{2 w}} \frac{u^{k}}{\left(1+u^{2}\right)^{s+1}} d u \\
& =-2 \int_{0}^{\infty} \frac{u^{k}}{\left(1+u^{2}\right)^{s+1}} d u=-B\left(\frac{k+1}{2}, s-\frac{k-1}{2}\right)
\end{aligned}
$$

where $B(x, y), x, y>0$, represents the Beta Euler's function.

By combining the above estimates we conclude that $\lim _{w \rightarrow 0} w H^{k}(w)=0$, when $k$ is odd. Assume now that $k$ is even. In this case we obtain that

$$
\begin{aligned}
\lim _{w \rightarrow 0^{+}} H^{k}(w) & =\sum_{s=1}^{\frac{k}{2}-1} c_{k-1, s, s, 0} \int_{0}^{1}\left(\log \frac{1}{r}\right)^{k-1} \frac{\left(1-r^{2}\right) r^{s-1}}{(1-r)^{2(s+1)}} d r \\
& -\sum_{s=\frac{k}{2}}^{k-1} c_{k-1, s, k-s-1,2 s-k+1} B\left(\frac{k+1}{2}, s-\frac{k-1}{2}\right) .
\end{aligned}
$$


By taking into account (8) and the duplication formula for the Gamma Euler's function, we can write

$$
\begin{aligned}
& \lim _{w \rightarrow 0^{+}} H^{k}(w)=-\sum_{s=\frac{k}{2}}^{k-1} \frac{(-1)^{s+1}(k-1) ! s !}{2^{k-2 s-1}(2 s-k+1) !(k-s-1) !} B\left(\frac{k+1}{2}, s-\frac{k-1}{2}\right) \\
& =\frac{\pi(\Gamma(k))^{2}}{2^{k-2} \Gamma\left(\frac{k}{2}\right)} \sum_{s=\frac{k}{2}}^{k-1} \frac{(-1)^{s}}{(2 s-k+1)(k-s-1) !\left(s-\frac{k}{2}\right) !}=\frac{(-1)^{\frac{k}{2}} \pi(\Gamma(k))^{2}}{2^{k-2}\left(\Gamma\left(\frac{k}{2}\right)\right)^{2}} \sum_{r=0}^{\frac{k}{2}-1}(-1)^{r}\left(\begin{array}{c}
\frac{k}{2}-1 \\
r
\end{array}\right) \frac{1}{2 r+1} \\
& =\frac{(-1)^{\frac{k}{2}} \pi(\Gamma(k))^{2}}{2^{k-2}\left(\Gamma\left(\frac{k}{2}\right)\right)^{2}} \int_{0}^{1}\left(1-t^{2}\right)^{\frac{k}{2}-1} d t=(-1)^{\frac{k}{2}} \pi \Gamma(k) .
\end{aligned}
$$

By proceeding as in Step 1 we can see that

$$
\begin{aligned}
& \frac{d^{k-1}}{d \theta^{k-1}} \int_{0}^{\pi} f(\varphi) \int_{0}^{1}\left(\log \frac{1}{r}\right)^{k-1}\left(\frac{1-r^{2}}{1-2 r \cos (\theta-\varphi)+r^{2}}-1\right) \frac{d r}{r} d \varphi \\
& (24) \quad=\int_{0}^{\pi} f(\varphi) \int_{0}^{1}\left(\log \frac{1}{r}\right)^{k-1} \frac{\partial^{k-1}}{\partial \theta^{k-1}}\left(\frac{1-r^{2}}{1-2 r \cos (\theta-\varphi)+r^{2}}-1\right) \frac{d r}{r} d \varphi, \quad \theta \in(0, \pi) .
\end{aligned}
$$

It is clear that

$$
\begin{aligned}
\frac{d^{k-1}}{d \theta^{k-1}} \int_{0}^{\pi} f(\varphi) \int_{0}^{1}\left(\log \frac{1}{r}\right)^{k-1} & \left(\frac{1-r^{2}}{1-2 r \cos (\theta-\varphi)+r^{2}}-1\right) \frac{d r}{r} d \varphi \\
& =\int_{-\pi}^{\pi} \tilde{f}(\varphi) H^{k}(\theta-\varphi) d \varphi, \quad \theta \in(0, \pi)
\end{aligned}
$$

where $\tilde{f}(\varphi)=\left\{\begin{array}{ll}f(\varphi), & \varphi \in[0, \pi) \\ 0, & \varphi \in(-\pi, 0)\end{array}\right.$, and $\tilde{f}(\varphi)=\tilde{f}(\varphi+2 \pi), \varphi \in \mathbb{R}$.

Also, since $H^{k} \in L^{1}(-\pi, \pi)$, we have that,

$$
\begin{aligned}
\frac{d}{d \theta} \int_{-\pi}^{\pi} \tilde{f}(\varphi) H^{k}(\theta-\varphi) d \varphi=\frac{\partial}{\partial \theta} \int_{-\pi}^{\pi} \tilde{f}(\theta-u) H^{k}(u) d u=\int_{-\pi}^{\pi} \frac{\partial}{\partial \theta} \tilde{f}(\theta-u) H^{k}(u) d u \\
=-\lim _{\varepsilon \rightarrow 0^{+}} \int_{-\pi,|u|>\varepsilon}^{\pi} \frac{d}{d u}[\tilde{f}(\theta-u)] H^{k}(u) d u \\
=-\lim _{\varepsilon \rightarrow 0^{+}}\left(\tilde{f}(\theta-u) H^{k}(u)\right]_{\varepsilon}^{\pi}-\int_{\varepsilon}^{\pi} \tilde{f}(\theta-u) \frac{d}{d u} H^{k}(u) d u \\
\left.\left.\quad+\tilde{f}(\theta-u) H^{k}(u)\right]_{-\pi}^{-\varepsilon}-\int_{-\pi}^{-\varepsilon} \tilde{f}(\theta-u) \frac{d}{d u} H^{k}(u) d u\right) \\
=-\lim _{\varepsilon \rightarrow 0^{+}}\left(\tilde{f}(\theta-\pi) H^{k}(\pi)-\tilde{f}(\theta-\varepsilon) H^{k}(\varepsilon)+\tilde{f}(\theta+\varepsilon) H^{k}(-\varepsilon)\right. \\
\left.\quad-\tilde{f}(\theta+\pi) H^{k}(-\pi)-\int_{-\pi,|u|>\varepsilon}^{\pi} \tilde{f}(\theta-u) \frac{d}{d u} H^{k}(u) d u\right), \quad \theta \in(0, \pi) .
\end{aligned}
$$


Since the function $H^{k}$ is even when $k$ is odd and $H^{k}$ is odd when $k$ is even, we conclude that

$$
\begin{aligned}
& \frac{d}{d \theta} \int_{0}^{\pi} f(\varphi) H^{k}(\theta-\varphi) d \varphi=\lim _{\varepsilon \rightarrow 0^{+}} \int_{-\pi,|\theta-\varphi|>\varepsilon}^{\pi} \tilde{f}(\varphi)\left(\frac{d}{d u} H^{k}\right)(\theta-\varphi) d \varphi \\
& \quad-\lim _{\varepsilon \rightarrow 0^{+}}(f(\theta+\varepsilon)-f(\theta-\varepsilon)) H^{k}(\varepsilon)=\lim _{\varepsilon \rightarrow 0^{+}} \int_{0,|\theta-\varphi|>\varepsilon}^{\pi} f(\varphi) \frac{\partial}{\partial \theta} H^{k}(\theta-\varphi) d \varphi, \quad \theta \in(0, \pi),
\end{aligned}
$$

when $k$ is odd, and

$$
\begin{array}{rl}
\frac{d}{d \theta} \int_{0}^{\pi} f & f(\varphi) H^{k}(\theta-\varphi) d \varphi \\
\quad & =\lim _{\varepsilon \rightarrow 0^{+}}(f(\theta+\varepsilon)+f(\theta-\varepsilon)) H^{k}(\varepsilon)+\lim _{\varepsilon \rightarrow 0^{+}} \int_{0,|\theta-\varphi|>\varepsilon}^{\pi} f(\varphi) \frac{\partial}{\partial \theta} H^{k}(\theta-\varphi) d \varphi \\
& =2 f(\theta)(-1)^{\frac{k}{2}} \pi \Gamma(k)+\lim _{\varepsilon \rightarrow 0^{+}} \int_{0,|\theta-\varphi|>\varepsilon}^{\pi} f(\varphi) \frac{\partial}{\partial \theta} H^{k}(\theta-\varphi) d \varphi, \quad \theta \in(0, \pi),
\end{array}
$$

when $k$ is even.

Step 4. We now finish the proof of Theorem 1.1. We firstly write, according to (4),

$$
\begin{aligned}
\frac{d^{k-1}}{d \theta^{k-1}} L_{\lambda}^{-\frac{k}{2}} f(\theta)= & \int_{0}^{\pi} f(\varphi) R_{\lambda}^{k, k-1}(\theta, \varphi) d m_{\lambda}(\varphi)=\int_{0}^{\pi} f(\varphi) \frac{R^{k, k-1}(\theta, \varphi)}{(\sin \theta \sin \varphi)^{\lambda}} d m_{\lambda}(\varphi) \\
& +\int_{0}^{\pi} f(\varphi)\left(R_{\lambda}^{k, k-1}(\theta, \varphi)-\frac{R^{k, k-1}(\theta, \varphi)}{(\sin \theta \sin \varphi)^{\lambda}}\right) d m_{\lambda}(\varphi), \quad \theta \in(0, \pi),
\end{aligned}
$$

where, for every $\theta, \varphi \in(0, \pi)$,

$$
R^{k, k-1}(\theta, \varphi)=\frac{1}{2 \pi \Gamma(k)} \int_{0}^{1}\left(\log \frac{1}{r}\right)^{k-1} \frac{\partial^{k-1}}{\partial \theta^{k-1}}\left(\frac{1-r^{2}}{1-2 r \cos (\theta-\varphi)+r^{2}}-1\right) \frac{d r}{r} .
$$

Moreover, by (23) and (24) we have, for every $\theta \in(0, \pi)$,

$$
\begin{aligned}
& \text { (25) } \frac{d}{d \theta} \int_{0}^{\pi} f(\varphi) \frac{R^{k, k-1}(\theta, \varphi)}{(\sin \theta \sin \varphi)^{\lambda}} d m_{\lambda}(\varphi)=\frac{1}{2 \pi \Gamma(k)} \frac{d}{d \theta}\left(\frac{1}{(\sin \theta)^{\lambda}}\right. \\
& \left.\quad \times \frac{d^{k-1}}{d \theta^{k-1}} \int_{0}^{\pi} \frac{f(\varphi)}{(\sin \varphi)^{\lambda}} \int_{0}^{1}\left(\log \frac{1}{r}\right)^{k-1}\left(\frac{1-r^{2}}{1-2 r \cos (\theta-\varphi)+r^{2}}-1\right) \frac{d r}{r} d m_{\lambda}(\varphi)\right) \\
& =-\frac{\lambda \cos \theta}{(\sin \theta)^{\lambda+1}} \int_{0}^{\pi} \frac{f(\varphi)}{(\sin \varphi)^{\lambda}} R^{k, k-1}(\theta, \varphi) d m_{\lambda}(\varphi)+\frac{1}{2 \pi \Gamma(k)} \frac{1}{(\sin \theta)^{\lambda}} \\
& \times \frac{d^{k}}{d \theta^{k}} \int_{0}^{\pi} \frac{f(\varphi)}{(\sin \varphi)^{\lambda}} \int_{0}^{1}\left(\log \frac{1}{r}\right)^{k-1}\left(\frac{1-r^{2}}{1-2 r \cos (\theta-\varphi)+r^{2}}-1\right) \frac{d r}{r} d m_{\lambda}(\varphi)
\end{aligned}
$$




$$
\begin{aligned}
= & -\frac{\lambda \cos \theta}{(\sin \theta)^{\lambda+1}} \int_{0}^{\pi} \frac{f(\varphi)}{(\sin \varphi)^{\lambda}} R^{k, k-1}(\theta, \varphi) d m_{\lambda}(\varphi) \\
& +\frac{1}{(\sin \theta)^{\lambda}} \lim _{\varepsilon \rightarrow 0^{+}} \int_{0,|\theta-\varphi|>\varepsilon}^{\pi} \frac{f(\varphi)}{(\sin \varphi)^{\lambda}} R^{k}(\theta, \varphi) d m_{\lambda}(\varphi)+\gamma_{k} f(\theta),
\end{aligned}
$$

where the integral after the last equal sign is absolutely convergent and $\gamma_{k}=0$, if $k$ is odd and $\gamma_{k}=(-1)^{\frac{k}{2}}$, when $k$ is even.

A careful study of Lemma 2.1 and again a distributional argument allow us to justify the differentiation under the integral sign (see Lemma 4.2 in Appendix) to get

$$
\begin{gathered}
\frac{d}{d \theta} \int_{0}^{\pi} f(\varphi)\left(R_{\lambda}^{k, k-1}(\theta, \varphi)-\frac{R^{k, k-1}(\theta, \varphi)}{(\sin \theta \sin \varphi)^{\lambda}}\right) d m_{\lambda}(\varphi)=\frac{\lambda \cos \theta}{(\sin \theta)^{\lambda+1}} \int_{0}^{\pi} \frac{f(\varphi)}{(\sin \varphi)^{\lambda}} R^{k, k-1}(\theta, \varphi) d m_{\lambda}(\varphi) \\
+\int_{0}^{\pi} f(\varphi)\left(R_{\lambda}^{k}(\theta, \varphi)-\frac{R^{k}(\theta, \varphi)}{(\sin \theta \sin \varphi)^{\lambda}}\right) d m_{\lambda}(\varphi), \quad \text { a.e. } \theta \in(0, \pi),
\end{gathered}
$$

where all the integrals are absolutely convergent.

By combining (25) and (26) we conclude that

$$
\begin{aligned}
& \frac{d^{k}}{d \theta^{k}} L_{\lambda}^{-\frac{k}{2}} f(\theta)=\frac{d}{d \theta} \int_{0}^{\pi} f(\varphi)\left(R_{\lambda}^{k, k-1}(\theta, \varphi)-\frac{R^{k, k-1}(\theta, \varphi)}{(\sin \theta \sin \varphi)^{\lambda}}\right) d m_{\lambda}(\varphi) \\
& \quad+\frac{d}{d \theta} \int_{0}^{\pi} f(\varphi) \frac{R^{k, k-1}(\theta, \varphi)}{(\sin \theta \sin \varphi)^{\lambda}} d m_{\lambda}(\varphi) \\
& =\lim _{\varepsilon \rightarrow 0^{+}} \int_{0,|\theta-\varphi|>\varepsilon}^{\pi} f(\varphi) R_{\lambda}^{k}(\theta, \varphi) d m_{\lambda}(\varphi)-\lim _{\varepsilon \rightarrow 0^{+}} \int_{0,|\theta-\varphi|>\varepsilon}^{\pi} f(\varphi) \frac{R^{k}(\theta, \varphi)}{(\sin \theta \sin \varphi)^{\lambda}} d m_{\lambda}(\varphi) \\
& \quad+\lambda \frac{\cos \theta}{(\sin \theta)^{\lambda+1}} \int_{0}^{\pi} \frac{f(\varphi)}{(\sin \varphi)^{\lambda}} R^{k, k-1}(\theta, \varphi) d m_{\lambda}(\varphi)+\frac{d}{d \theta} \int_{0}^{\pi} f(\varphi) \frac{R^{k, k-1}(\theta, \varphi)}{(\sin \theta \sin \varphi)^{\lambda}} d m_{\lambda}(\varphi) \\
& =\lim _{\varepsilon \rightarrow 0^{+}} \int_{0,|\theta-\varphi|>\varepsilon}^{\pi} f(\varphi) R_{\lambda}^{k}(\theta, \varphi) d m_{\lambda}(\varphi)+\gamma_{k} f(\theta), \quad \text { a.e. } \theta \in(0, \pi) .
\end{aligned}
$$

Thus the proof of Theorem 1.1 is complete.

\section{Proof of Theorem 1.2}

In order to show Theorem 1.2 we need to improve Lemma 2.1 as follows,

Lemma 3.1. Let $k \in \mathbb{N}$. If $R^{k}$ and $A_{2}$ are defined as in Lemma 2.1, then

$$
R^{k}(\theta, \varphi)=M_{k} \frac{1}{\sin (\theta-\varphi)}+O\left(\sqrt{\frac{1}{|\theta-\varphi|}}\right), \quad(\theta, \varphi) \in A_{2}, \quad \theta \neq \varphi,
$$

for a certain $M_{k} \in \mathbb{R}$. Moreover, $M_{k}=0$ provided that $k$ is even. 
Proof. According to (6) with $\lambda=t=0$ we have that

$$
\frac{\partial}{\partial \theta^{k}}\left(\frac{1}{\Delta_{r}}\right)=\sum_{s, i, j} c_{k, s, i, j} \frac{r^{i+j} a^{i} b^{j}}{\Delta_{r}^{1+s}}
$$

where $a=\cos (\theta-\varphi), b=-\sin (\theta-\varphi)$, and $c_{k, s, i, j} \neq 0$ only if $s=1, \ldots, k, j \geq 2 s-k$ and $i+j=s$.

Note firstly that

$$
\int_{0}^{\frac{1}{2}}\left(\log \frac{1}{r}\right)^{k-1}\left(1-r^{2}\right)\left|\frac{\partial}{\partial \theta^{k}}\left(\frac{1}{1+r^{2}-2 r \cos (\theta-\varphi)}\right)\right| \frac{d r}{r} \leq C, \quad \theta, \varphi \in(0, \pi) .
$$

Also, we have

$$
\begin{aligned}
\int_{\frac{1}{2}}^{1}\left(\log \frac{1}{r}\right)^{k-1}\left(1-r^{2}\right) \frac{r^{i+j}|a|^{i}|b|^{j}}{\Delta_{r}^{1+s}} \frac{d r}{r} & \leq C \int_{\frac{1}{2}}^{1} \frac{(1-r)^{k}|b|^{j}}{\left((1-r)^{2}+\Delta\right)^{1+s}} d r \\
& \leq C \frac{|b|^{j}}{\Delta^{s-\frac{k}{2}+\frac{1}{2}}} \int_{0}^{\frac{1}{2 \sqrt{\Delta}}} \frac{u^{k}}{(1+u)^{2+2 s}} d u \\
& \leq C \frac{|b|^{j}}{\Delta^{\frac{j}{2}+\frac{1}{4}}} \int_{0}^{\frac{1}{2 \sqrt{\Delta}}} \frac{u^{2 s-j+\frac{1}{2}}}{(1+u)^{2+2 s}} d u \\
& \leq C \frac{1}{\sqrt{|\theta-\varphi|}}, \quad(\theta, \varphi) \in A_{2}, \quad \theta \neq \varphi
\end{aligned}
$$

provided that $s=1, \ldots, k, i+j=s, j>2 s-k$.

Assume now $s=1, \ldots, k, i+j=s, j=2 s-k$. By using the mean value theorem we get

$$
\begin{aligned}
\int_{\frac{1}{2}}^{1}\left(\log \frac{1}{r}\right)^{k-1}(1 & \left.-r^{2}\right) \frac{r^{i+j} a^{i} b^{j}}{\Delta_{r}^{1+s}} \frac{d r}{r} \\
& =2 \int_{\frac{1}{2}}^{1} \frac{(1-r)^{k}}{\left((1-r)^{2}+\Delta\right)^{1+s}} d r a^{i} b^{j}+O\left(\frac{1}{\sqrt{|\theta-\varphi|}}\right), \quad \theta, \varphi \in A_{2}, \quad \theta \neq \varphi .
\end{aligned}
$$

Moreover, since $2 s-k \geq 0$,

$$
\int_{\frac{1}{2}}^{1} \frac{(1-r)^{k}}{\left((1-r)^{2}+\Delta\right)^{1+s}} d r=\frac{1}{\Delta^{s-\frac{k}{2}+\frac{1}{2}}}\left(\int_{0}^{\infty} \frac{u^{k} d u}{\left(1+u^{2}\right)^{1+s}}-\int_{\frac{1}{2 \sqrt{\Delta}}}^{\infty} \frac{u^{k} d u}{\left(1+u^{2}\right)^{1+s}}\right)
$$

and

$$
\begin{aligned}
\frac{|a|^{i}|b|^{j}}{\Delta^{s-\frac{k}{2}+\frac{1}{2}}} \int_{\frac{1}{2 \sqrt{\Delta}}}^{\infty} \frac{u^{k} d u}{\left(1+u^{2}\right)^{1+s}} & \leq C \frac{1}{\Delta^{1 / 4}} \int_{\frac{1}{2 \sqrt{\Delta}}}^{\infty} \frac{u^{k+\frac{1}{2}} d u}{(1+u)^{2+s}} \\
& \leq \frac{C}{\Delta^{\frac{1}{4}}} \leq \frac{C}{|\theta-\varphi|^{\frac{1}{2}}}, \quad \theta, \varphi \in A_{2}, \theta \neq \varphi .
\end{aligned}
$$


Also, if $k$ is odd, we have that

$\frac{a^{i} b^{j}}{\Delta^{s-\frac{k}{2}+\frac{1}{2}}}=\frac{(\cos (\theta-\varphi))^{i}(-\sin (\theta-\varphi))^{j}}{(2(1-\cos (\theta-\varphi)))^{\frac{j}{2}+\frac{1}{2}}}=-\frac{1}{\sin (\theta-\varphi)}+O\left(\frac{1}{|\theta-\varphi|^{\frac{1}{2}}}\right), \quad \theta, \varphi \in A_{2}, \theta \neq \varphi$.

By combining the above estimates we conclude that

$$
R^{k}(\theta, \varphi)=M_{k} \frac{1}{\sin (\theta-\varphi)}+O\left(\frac{1}{|\theta-\varphi|^{\frac{1}{2}}}\right), \quad \theta, \varphi \in A_{2}, \theta \neq \varphi
$$

for a certain $M_{k} \in \mathbb{R}$, for every $k$ odd.

Assume now that $k$ is even. We get

$$
\frac{a^{i} b^{j}}{\Delta^{s-\frac{k}{2}+\frac{1}{2}}}=\frac{1}{|\sin (\theta-\varphi)|}+O\left(\frac{1}{|\theta-\varphi|^{\frac{1}{2}}}\right), \quad \theta, \varphi \in A_{2}, \theta \neq \varphi .
$$

Hence, from Lemma 2.1 we deduce that, for every $\theta, \varphi \in A_{2}, \theta \neq \varphi$,

$$
R_{\lambda}^{k}(\theta, \varphi)=M_{k} \frac{1}{|\sin (\theta-\varphi)|(\sin \theta \sin \varphi)^{\lambda}}+O\left(\frac{1}{(\sin \theta \sin \varphi)^{\lambda+1 / 2}}\left(1+\sqrt{\frac{\sin \theta}{|\theta-\varphi|}}\right)\right) .
$$

By virtue of Theorem 1.1, $M_{k}=0$ because

$$
\lim _{\varepsilon \rightarrow 0^{+}} \int_{\theta / 2,|\theta-\varphi|>\varepsilon}^{3 \theta / 2} \frac{1}{|\sin (\theta-\varphi)|}(\sin \varphi)^{\lambda} d \varphi
$$

does not exist for every $\theta \in(0, \pi / 2)$.

From Lemmas 2.1 and 3.1 we deduce that,

$$
R_{\lambda}^{k}(\theta, \varphi)=\left\{\begin{array}{l}
O\left((\sin \varphi)^{-(2 \lambda+1)}\right), \quad(\theta, \varphi) \in A_{1} \\
\frac{M_{k}}{(\sin \theta \sin \varphi)^{\lambda} \sin (\theta-\varphi)}+O\left(\frac{1}{(\sin \varphi)^{2 \lambda+1}}\left(1+\sqrt{\frac{\sin \varphi}{|\theta-\varphi|}}\right)\right),(\theta, \varphi) \in A_{2}, \theta \neq \varphi ; \\
O\left((\sin \theta)^{-(2 \lambda+1)}\right), \quad(\theta, \varphi) \in A_{3} .
\end{array}\right.
$$

By using (27) we can prove Theorem 1.2 by proceeding as in the proof of [1, Proposition 8.1].

\section{Appendix}

In this appendix we present the results we need about differentiation under the integral sign. We think that these results are wellknown but we have not found a exact reference (only the unpublished notes [5]). Then we prefer to include here a proof of the result in the form we use. We look for conditions on a function $f$ defined on $\mathbb{R} \times \mathbb{R}$ in order that the formula

$$
\frac{\partial}{\partial x} \int_{\mathbb{R}} f(x, y) d y=\int_{\mathbb{R}} \frac{\partial}{\partial x} f(x, y) d y, \quad \text { a.e. } x \in \mathbb{R},
$$


holds.

In the following we establish conditions on a function $f$ in order that distributional and classical derivatives of $f$ coincide.

Lemma 4.1. Let $-\infty \leq a<b \leq+\infty$. Assume that $f$ is a continuous function on $I \times I$, where $I=(a, b)$, such that

(i) For every $y \in I$, the function $\frac{\partial}{\partial x} f(x, y) d y$ is continuous on $I \backslash\{y\}$, where the derivative is understood in the classical sense.

(ii) For every $y \in I$ and every compact subset $K$ of $I, \int_{K}|f(x, y)| d x<+\infty$, and

$$
\int_{K}\left|\frac{\partial f}{\partial x}(x, y)\right| d x<+\infty
$$

Then, $D_{x} f(x, y)=\frac{\partial}{\partial x} f(x, y)$, for every $y \in I$. Here, as above, $D_{x} f(x, y)$ denotes the distributional derivative respect to $x$ of $f$.

Proof. Let $g \in C_{c}^{\infty}(I)$. We can write

$$
\begin{aligned}
& <D_{x} f(x, y), g(x)>=-\lim _{\varepsilon \rightarrow 0^{+}}\left(\int_{a}^{y-\varepsilon}+\int_{y+\varepsilon}^{b}\right) g^{\prime}(x) f(x, y) d x \\
& \quad=\lim _{\varepsilon \rightarrow 0^{+}}\left[-g(y-\varepsilon) f(y-\varepsilon, y)+g(y+\varepsilon) f(y+\varepsilon, y)+\left(\int_{a}^{y-\varepsilon}+\int_{y+\varepsilon}^{b}\right) g(x) \frac{\partial f}{\partial x}(x, y) d x\right] \\
& =\int_{a}^{b} g(x) \frac{\partial f}{\partial x}(x, y) d x, \quad y \in I .
\end{aligned}
$$

Then, $D_{x} f(x, y)=\frac{\partial f}{\partial x}(x, y), y \in I$.

The differentiations under the integral sign that we have made in the proof of our results can be justified by using the following one.

Lemma 4.2. Suppose that $f$ is a measurable function defined on $\mathbb{R} \times \mathbb{R}$ that satisfies the following conditions:

(i) for every compact subset $K$ of $\mathbb{R}, \int_{K} \int_{\mathbb{R}}|f(x, y)| d y d x<\infty$, and

(ii) there exists a measurable function $g$ on $\mathbb{R} \times \mathbb{R}$ such that $\int_{K} \int_{\mathbb{R}}|g(x, y)| d y d x<\infty$, for every compact subset $K$ of $\mathbb{R}$, and that the distributional derivative $D_{x} f(\cdot, y)$ is represented by $g(\cdot, y)$, for every $y \in \mathbb{R}$.

Then,

$$
\frac{\partial}{\partial x} \int_{\mathbb{R}} f(x, y) d y=\int_{\mathbb{R}} \frac{\partial}{\partial x} f(x, y) d y, \quad \text { a.e. } x \in \mathbb{R}
$$


where the derivatives are understood in the classical sense.

Proof. We define the function $h(x)=\int_{\mathbb{R}} f(x, y) d y, x \in \mathbb{R}$. By (i) $h$ defines a regular distribution that we continue denoting by $h$. According to [12, Chap. 2, $\S 5$, Theorem V], we have that

$$
\frac{\partial}{\partial x} f(x, y)=g(x, y), \quad \text { a.e. }(x, y) \in \mathbb{R} \times \mathbb{R},
$$

where the derivative is understood in the classical sense. Moreover, if $F \in C_{c}^{\infty}(\mathbb{R})$, then

$$
<D_{x} h, F>=\int_{\mathbb{R}} F(x) \int_{\mathbb{R}} \frac{\partial f}{\partial x}(x, y) d y d x .
$$

Hence, $D_{x} h(x)=\int_{\mathbb{R}} \frac{\partial f}{\partial x}(x, y) d y$ in the distributional sense. By using again [12, Chap. 2, $\S 5$, Theorem V] we conclude that

$$
\frac{\partial}{\partial x} h(x)=\int_{\mathbb{R}} \frac{\partial}{\partial x} f(x, y) d y, \quad \text { a.e. } x \in \mathbb{R} .
$$

Thus the proof is completed.

\section{REFERENCES}

[1] D. Buraczewski, T. Martínez, J. L. Torrea, and R. Urban, On the Riesz transform associated with the ultraspherical polynomials, J. Anal. Math. 98 (2006), 113-143.

[2] D. Buraczewski, T. Martínez, and J. L. Torrea, Calderón-Zygmund operators associated to ultraspherical expansions, Canad. J. Math. 59 (2007), no. 6, 1223-1244.

[3] J. T. Campbell, R. L. Jones, K. Reinhold, and M. Wierdl, Oscillation and variation for the Hilbert transform, Duke Math. J. 105 (2000), no. 1, 59-83.

[4] J. T. Campbell, R. L. Jones, K. Reinhold, and M. Wierdl, Oscillation and variation for singular integrals in higher dimensions, Trans. Amer. Math. Soc. 355 (2003), no. 5, 2115-2137.

[5] S. Cheng, Differentiation under the integral sign using weak derivatives, unpublished manuscript. http://www.gold-saucer.org/math

[6] T. A. Gillespie and J. L. Torrea, Dimension free estimates for the oscillation of Riesz transforms, Israel J. Math. 141 (2004), 125-144.

[7] L. Grafakos, Classical and modern Fourier analysis, Pearson Education, Inc., Upper Saddle River, N.J., 2004.

[8] R. L. Jones and G. Wang, Variation inequalities for the Fejér and Poisson kernels, Trans. Amer. Math. Soc. 356 (2004), no. 11, 4493-4518.

[9] B. Muckenhoupt, Transplantation theorems and multiplier theorems for Jacobi series, Mem. Amer. Math. Soc. 64 (1986), no. 356.

[10] B. Muckenhoupt and E. M. Stein, Classical expansions and their relation to conjugate harmonic functions, Trans. Amer. Math. Soc. 118 (1965), 17-92. 
[11] S. Roman, The formula of Fà̀ di Bruno, Amer. Math. Monthly 87 (1980), 805-809.

[12] L. Schwartz, Théorie des distributions, Hermann, París, 1973.

[13] E. M. Stein, Topics in harmonic analysis related to the Littlewood-Paley theory., Annals of Mathematics Studies, No. 63, Princeton University Press, Princeton, N.J., 1970.

[14] G. Szegő, Orthogonal polynomials, fourth ed., American Mathematical Society, Providence, R.I., 1975, American Mathematical Society, Colloquium Publications, Vol. XXIII.

Departamento de Análisis Matemático, Universidad de la laguna, Campus de Anchieta, Avda. Astrofísico Francisco Sánchez, s/n, 38271 La Laguna (Sta. Cruz de Tenerife), Spain

E-mail address: jbetanco@ull.es; jcfarina@ull.es; lrguez@ull.es

Departamento de Matemática, Universidad Nacional del Sur, Avda. Alem 1253 - 2 Piso, 8000 Bahía Blanca, Buenos Aires, Argentina

E-mail address: ricardo.testoni@uns.edu.ar 